\title{
Global genomic analysis reveals rapid control of a robust innate response in SIV-infected sooty mangabeys
}

Steven E. Bosinger, ${ }^{1,2}$ Qingsheng Li, ${ }^{3}$ Shari N. Gordon, ${ }^{1}$ Nichole R. Klatt, ${ }^{1}$ Lijie Duan, ${ }^{3}$ Luoling Xu, ${ }^{2}$ Nicholas Francella, ${ }^{1}$ Abubaker Sidahmed, ${ }^{2}$ Anthony J. Smith, ${ }^{3}$

Elizabeth M. Cramer, ${ }^{1}$ Ming Zeng, ${ }^{3}$ David Masopust, ${ }^{3}$ John V. Carlis, ${ }^{4}$ Longsi Ran, ${ }^{2}$ Thomas H. Vanderford, ${ }^{1}$ Mirko Paiardini, ${ }^{1}$ R. Benjamin Isett, ${ }^{5}$ Don A. Baldwin, ${ }^{1,5}$ James G. Else, ${ }^{6}$ Silvija I. Staprans, ${ }^{7}$ Guido Silvestri, ${ }^{1,6}$ Ashley T. Haase, ${ }^{3}$ and David J. Kelvin ${ }^{2,8}$

1Department of Pathology and Laboratory Medicine, and Microbiology, University of Pennsylvania School of Medicine, Philadelphia, Pennsylvania, USA.

${ }^{2}$ Division of Experimental Therapeutics, University Health Network, Toronto, Ontario, Canada. ${ }^{3}$ Department of Microbiology, Medical School, University of Minnesota, Minneapolis, Minnesota, USA. ${ }^{4}$ Department of Computer Science and Engineering, Institute of Technology, University of Minnesota, Minneapolis, Minnesota, USA. ${ }^{5}$ Penn Microarray Facility, University of Pennsylvania School of Medicine, Philadelphia, Pennsylvania, USA. ${ }^{6}$ Yerkes National Primate Research Center, Emory University, Atlanta, Georgia, USA.

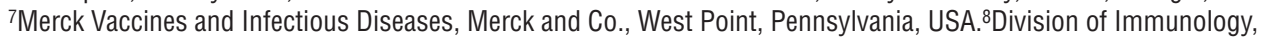

International Institute of Infection and Immunity, Shantou University Medical College, Shantou, Guangdong, People's Republic of China.

\begin{abstract}
Natural SIV infection of sooty mangabeys (SMs) is nonprogressive despite chronic virus replication. Strikingly, it is characterized by low levels of immune activation, while pathogenic SIV infection of rhesus macaques (RMs) is associated with chronic immune activation. To elucidate the mechanisms underlying this intriguing phenotype, we used high-density oligonucleotide microarrays to longitudinally assess host gene expression in SIV-infected SMs and RMs. We found that acute SIV infection of SMs was consistently associated with a robust innate immune response, including widespread upregulation of IFN-stimulated genes (ISGs) in blood and lymph nodes. While SMs exhibited a rapid resolution of ISG expression and immune activation, both responses were observed chronically in RMs. Systems biology analysis indicated that expression of the lymphocyte inhibitory receptor LAG3, a marker of T cell exhaustion, correlated with immune activation in SIV-infected RMs but not SMs. Our findings suggest that active immune regulatory mechanisms, rather than intrinsically attenuated innate immune responses, underlie the low levels of immune activation characteristic of SMs chronically infected with SIV.
\end{abstract}

\section{Introduction}

HIV infection is characterized by progressive depletion of $\mathrm{CD}^{+} \mathrm{T}$ cells that eventually results in clinically significant immunodeficiency. Although development of AIDS occurs years after primary HIV infection, it is now apparent that immune dysregulation occurs within the first few weeks of infection (1). While the level of virus replication clearly predicts the risk of developing AIDS in HIV-infected individuals (2), HIV disease progression is also characterized by a state of chronic, generalized immune activation that is thought to play a role in the pathogenesis of the immunodeficiency (3, 4). HIV/SIVassociated immune activation is characterized by (a) increased frequencies of $\mathrm{CD}^{+}$and $\mathrm{CD}^{+} \mathrm{T}$ cells expressing activation/proliferation markers; (b) accelerated turnover of $\mathrm{T}$ and $\mathrm{B}$ lymphocytes and NK cells; (c) expansion of T cells showing an effector phenotype; and (d) elevated serum levels of proinflammatory cytokines (5-8). The frequencies of $\mathrm{CD}^{+}$and $\mathrm{CD} 8^{+} \mathrm{T}$ cells with an activated phenotype are highly specific prognostic indicators of $\mathrm{CD} 4^{+} \mathrm{T}$ cell decline (9-11), progression to AIDS $(12,13)$, and patient survival (14).

In striking contrast to pathogenic HIV and SIV infections of humans and rhesus macaques (RMs) (Macaca mulatta), respectively, SIVsmm infection of sooty mangabeys (SMs) (Cercocebus atys),

Authorship note: Steven E. Bosinger and Qingsheng Li, as well as Guido Silvestri, Ashley T. Haase, and David J. Kelvin, contributed equally to this work

Conflict of interest: The authors have declared that no conflict of interest exists. Citation for this article: J. Clin. Invest. 119:3556-3572 (2009). doi:10.1172/JCI40115. an African monkey species that is a natural host for the virus, is typically nonpathogenic despite similarly high levels of virus replication (reviewed in ref. 15). This observation is quite intriguing since SIVsmm is the origin of both HIV-2 and the SIVmac strains that are highly pathogenic in RMs (16). Extensive characterization of SIV infection of SMs has shown that these infections share several features with pathogenic HIV/ SIV infection, namely, high virus replication, short life span of infected cells in vivo, tropism for $\mathrm{CD}^{+} \mathrm{T}$ cells, and depletion of mucosal CD4 ${ }^{+} \mathrm{T}$ cells (17-22). Several studies have demonstrated that a consistent feature of SIV-infected SMs, as well as other natural hosts for SIV, is the presence of low levels of immune activation during the chronic phase of infection (23). Various mechanisms have been proposed to explain this divergent phenotype between pathogenic and nonpathogenic infections, including the downregulation of the CD3-TCR complex by $\operatorname{SIV}$ Nef $(24,25)$, absence of microbial translocation from the intestinal lumen to the systemic circulation (26), reduced expression of CCR5 on $\mathrm{CD}^{+} \mathrm{T}$ cells with consequent decreased response of these cells to $\beta$-chemokine-mediated inflammatory stimuli (27), rapid upregulation of PD1 on the surface of $\mathrm{LN}$-derived $\mathrm{CD}^{+} \mathrm{T}$ cells (28), and reduced production of IFN- $\alpha$ by plasmacytoid DCs (pDCs) (29). At this time, the relative in vivo contribution of these mechanisms is still unknown. In addition, it is also unclear whether and to what extent, in SIVinfected SMs, the lack of chronic immune activation reflects an 


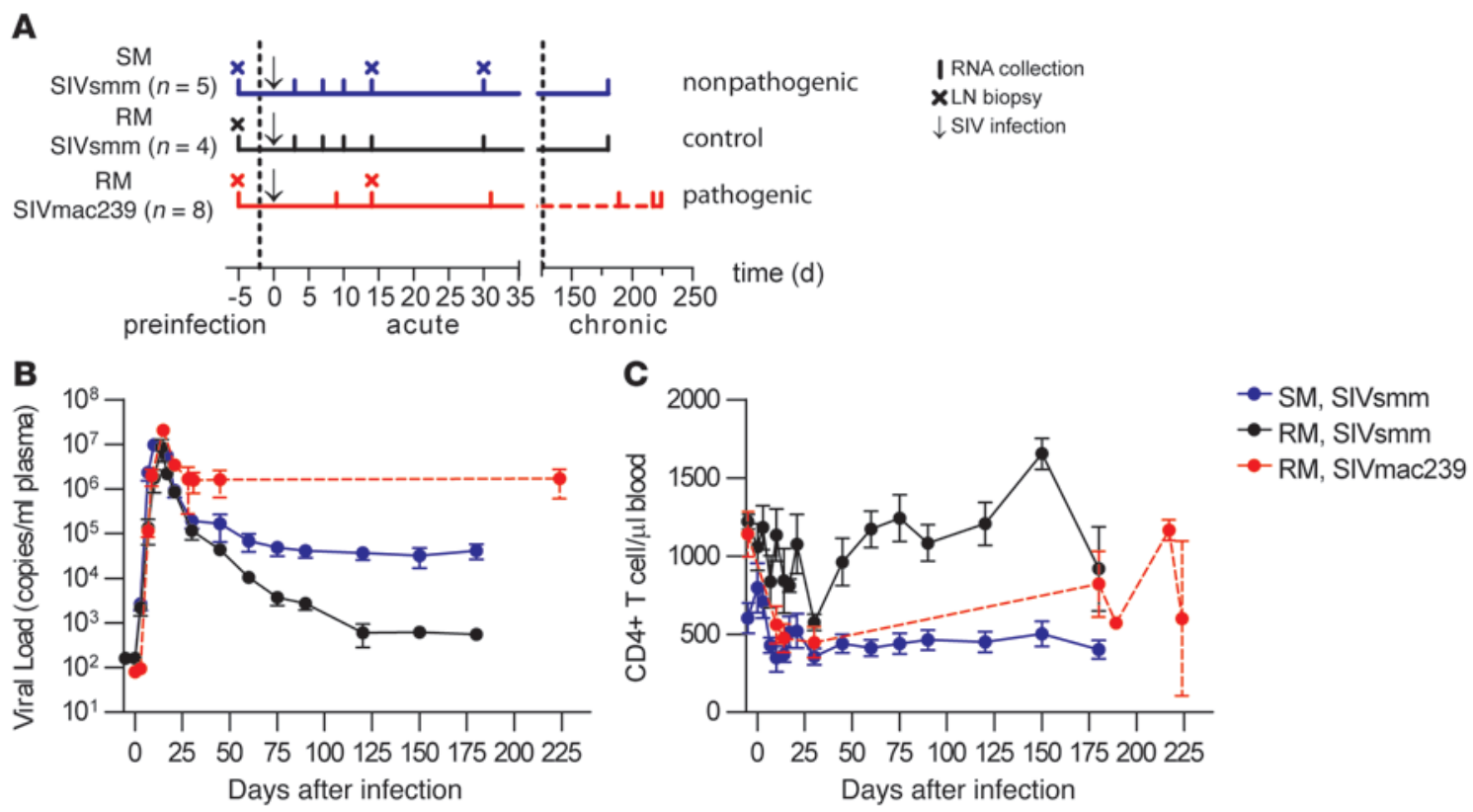

\section{Figure 1}

Experimental design, viral load, and CD4+ T cell kinetics in SIV-infected SMs and RMs. (A) Comparison of transcriptional profiles in peripheral blood induced by SIV infection was conducted in 5 SMs infected with SIVsmm to represent a nonpathogenic infection; 4 RMs inoculated with SIVsmm to compare infection of a nonnatural host with an isogenic virus; and 8 RMs infected with SIVmac239 to represent a classical, pathogenic infection. Arrows indicate SIV infection, vertical lines indicate RNA sampling time points, and crosses indicate LN biopsy time points. SIVsmminfected SMs (blue line) and RMs (black line) were sampled at days $-5,3,7,10,14$, and 30 (preinfection and acute) and 180 (chronic) after infection; SIVmac239-infected RMs (red line) were sampled at days -35, 9, 14, and 31 (preinfection and acute) and days 184-224 (chronic) after infection and were treated with ART and OKT8F mAb after day 50 after infection (see text and Methods for details), as indicated by the dashed line. During the chronic phase of infection in the SIVmac239 group, samples were collected at least 30 days after the last in vivo manipulation (i.e., ART and CD8+ lymphocyte depletion). (B) Longitudinal assessment of plasma viral load (RNA copies/ml plasma) in SMs $(n=5)$ and RMs $(n=4)$ infected with SIVsmm and RMs $(n=8)$ infected with SIVmac239. (C) Longitudinal analysis of the absolute numbers of CD3+CD4+ T lymphocytes per $\mathrm{ml}$ in peripheral blood, determined by flow cytometry. In B and C, the $x$ axis shows time after SIV infection, and error bars indicate SEM.

attenuated immune response to $\operatorname{SIV}(29,30)$ as opposed to rapid and active downmodulation of an otherwise strong antiviral immune response $(4,18,28,31)$.

To gain insight into the molecular mechanisms responsible for the divergent levels of immune activation and, ultimately, the different clinical outcome of SIV infections in RMs and SMs, we conducted a comparative, longitudinal assessment of gene expression in peripheral blood leukocytes and LNs using high-density oligonucleotide microarrays. We measured the transcriptional host response to SIV infection in 5 SMs and 4 RMs infected with uncloned isogenic SIVsmm, as well as 8 RMs infected with SIVmac239, at various time points during acute and chronic infection (i.e., day 3 to 180 after infection). We found that acute SIV infection of both RMs and SMs was consistently associated with robust immune activation and massive upregulation of IFN-stimulated genes (ISGs) in both blood and LNs, indicating a similarly strong innate immune response to the virus in both species and across different immunological compartments. However, this immune activation and ISG upregulation is transient in SMs but persistent in SIVmac239-infected RMs, suggesting that active immune regulatory mechanisms, rather than absence of innate immune responses, could underlie the low immune activation of chronically SIV-infected SMs. Finally, using a systems biology approach, we identified candidate genes whose upregulation may be responsible for the chronic immune activation of SIVmac239-infected RMs.

\section{Results}

Study design. To gain insight into the mechanisms underlying the divergent outcomes of SIV infection in SMs and RMs, both in terms of disease progression and overall immune activation, we conducted a comparative, longitudinal assessment of the gene-expression profiles during SIV infection in 3 cohorts of nonhuman primates. The study design is summarized in Figure 1A and involved the following: (a) 5 SMs infected with an uncloned SIVsmm as a model of nonpathogenic infection; (b) 5 RMs infected with the same stock of uncloned SIVsmm to compare gene expression between host species infected with an identical inoculum; and (c) 8 RMs infected with the prototypic pathogenic SIVmac239 clone, which represented an idealized model of progressive infection. In all animals, RNA from whole blood and LNs was collected prior to infection and at several intervals during acute and chronic SIV infection (Figure 1A). As part of another study, all macaques in the SIVmac239-infected group were treated with antiretroviral therapy for 28 days between day 57 and 85 after infection, with 4 of these animals also undergoing a 3 -day administration of the $\mathrm{CD}^{+}$lymphocyte-depleting antibody OKT8F during the antiretroviral treatment (Figure 1A). It is important to note, however, that these additional interventions were all conducted after the acute phase of infection and that the samples representing the chronic phase of infection were collected after the animals had been allowed to equilibrate for more than 30 days after both antiretroviral and $\mathrm{CD}^{+}$lymphocyte-depleting treatments. 
A

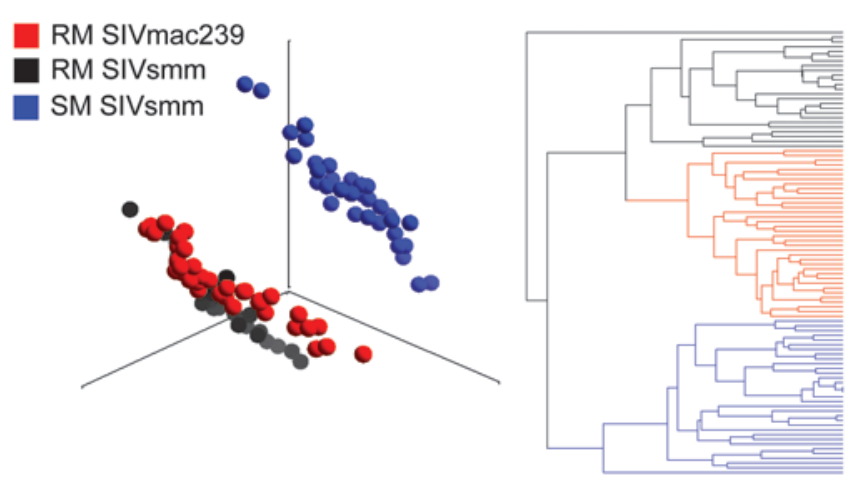

Figure 2

Divergence in the transcriptome of SMs and RMs. (A) PCA of complete gene-expression profiles measured on each individual array. PCA was performed on the $\log _{10}$-transformed, RMA-normalized intensities on individual arrays (52,024 probe sets per array) using a covariance dispersion matrix and normalized eigenvector scaling. Principal component (PC) no. 1 (37.4\%), PC no. 2 (11.7\%), and PC no. 3 (7.8\%) accounted for $56.9 \%$ of the variance in the data. Each colored circle indicates complete expression profiles of individual samples, with similarity between data sets displayed as proximity in 3D space (SIVsmminfected SMs, blue circles; SIVsmm-infected-RMs, black circles; SIVmac239-infected RMs, red circles). (B) Hierarchical clustering of individual array data sets was performed using a Euclidean metric and average linkage to determine distance between data sets and clusters, respectively. In $\mathbf{A}$ and $\mathbf{B}$, data sets from the 3 infection groups are indicated by color: SIVsmm-infected SMs (blue), SIVsmm-infected RMs (black), and SIVmac239-infected RMs (red).

Virological and immunological parameters of SIV infection of SMs and $R M s$. A productive SIVsmm infection was established in all SMs, with an average peak plasma viremia at day 10 after infection of $9.5 \times 10^{6}$ copies $/ \mathrm{ml}$ and mean viral set point, assessed at day 120 after infection, of $3.7 \times 10^{4} \mathrm{RNA}$ copies/ml (Figure $1 \mathrm{~A}$ ). In RMs inoculated with SIVsmm, an average peak plasma viremia similar to that of SMs $\left(8.5 \times 10^{6}\right.$ copies $\left./ \mathrm{ml}\right)$ was observed in 4 of 5 macaques at day 10 after infection (Figure 1B). However, viral load quickly decreased and reached a set point at day 120 after infection of approximately 600 copies/ml. In the fifth SIVsmm-infected RM, viral load was undetectable throughout the study except for a lowlevel blip of viral load ( $<530$ copies $/ \mathrm{ml}$ ) that was observed at day 21 after infection (data not shown). Due to the absence of robust virus replication, this animal was removed from further analyses. The SIVmac239-infected RMs showed a typical peak viremia (mean $2.1 \times 10^{7}$ copies $/ \mathrm{ml}$ at day 10 after infection) followed by a set-point level of $1.6 \times 10^{6} \mathrm{RNA}$ copies $/ \mathrm{ml}$ at day 30 after infection that remained constant at day $45\left(1.6 \times 10^{6} \mathrm{RNA}\right.$ copies $\left./ \mathrm{ml}\right)$ and at day $224\left(1.7 \times 10^{6} \mathrm{RNA}\right.$ copies/ml) after infection (Figure $\left.1 \mathrm{~B}\right)$.

As expected based on previous studies $(18,30,32)$, the absolute $\mathrm{CD}^{+} \mathrm{T}$ cell count in peripheral blood of the SMs was not substantially decreased by SIVsmm infection $($ range $=351-796 \mathrm{cells} / \mu \mathrm{l})$ over the course of the experiment (Figure 1C), confirming that our SM cohort was highly representative of the typical nonprogressive course of SIV infection in this natural host species. Of note, the SIVsmm-infected RMs also failed to show a substantial depletion of peripheral blood $\mathrm{CD}^{+} \mathrm{T}$ cells, with levels ranging between 575 and 1654 cells/ $\mu$ l over the course of the experiment (Figure $1 \mathrm{C})$. The relatively low viral load and absence of $\mathrm{CD} 4^{+}$lymphocyte depletion suggested that the SIVsmm-infected RMs did not represent a typical model of pathogenic SIV infection. This consideration provided the rationale to include in this study a group of SIVmac239-infected RMs, in which a rapid decline of CD $4^{+} \mathrm{T}$ cells was observed (Figure 1C). Based on the observed levels of virus replication and $\mathrm{CD}^{+} \mathrm{T}$ cell counts in SIVmac239-infected RMs, SIVsmm-infected RMs, and SIVsmm-infected SMs, we reasoned that these 3 cohorts were representative models of the progressive and nonprogressive outcomes of SIV infection. Based on these data, this study design would be suitable for comprehensive assessment of the profile of gene expression associated with the divergent course of infection.

Characterization of host transcriptomes of RMs and SMs. To compare gene-expression profiles during SIV infection from the 3 groups of nonhuman primates and thus identify a molecular signature of progressive versus nonprogressive outcome, we hybridized RNA derived from whole blood on Affymetrix RM 3' IVT expression arrays. Extensive characterization of the ability of the array to accurately quantify SM transcripts was performed and is detailed in the Supplemental Methods (Supplemental Figure 1 and Supplemental Tables 1 and 2; supplemental material available online with this article; doi:10.1172/JCI40115DS1). Hybridization of RNA derived from whole blood on Affymetrix arrays has been reported to suffer from nonspecific binding of hemoglobin transcripts; to improve signal detection we synthesized and utilized nonhuman primate hemoglobin consensus sequence-blocking oligonucleotides (Supplemental Table 3).

Principal component analysis (PCA) was used to reduce dimensionality and visualize the transcriptome on each individual array (Figure 2A). The global transcript profiles of SM samples were clearly distinguishable from RM samples. The transcriptional profiles of RM samples infected with SIVsmm or SIVmac239 were very similar although distinguishable, demonstrating that differences in the viral inocula affect gene expression. Similarly, unsupervised hierarchical clustering demonstrated that the 2 cohorts of SIV-infected RMs were more closely related to each other in their gene-expression profile than to the cohort of SIV-infected SMs (Figure 2B). The global visualization of gene-expression profiles demonstrated that large differences exist between species at the transcript level and suggested that while gene expression would differ between species due to SIV infection, a proportion of differential expression would also be due to interspecies variation or differences in hybridization efficacy.

Overview of the changes in gene expression induced by SIVsmm infection in SMs. To identify the molecular signature of SIV infection in SMs, we first longitudinally examined the gene-expression profile in the blood of these animals. Genes with differential expression were identified using stringent criteria: a significant change over time as determined by ANOVA (Benjamini-Hochberg corrected $P$ value $<0.0008342$ ) and \pm \pm 2 -fold or greater average change at 1 or more sampling intervals (detailed description of analysis in Supplemental Methods). Using this definition, 428 individual probe sets were identified as having significantly different transcription (Figure 3 and Supplemental Table 4). Hierarchical clustering of these 428 transcripts clearly demonstrated a quite dramatic effect of SIVsmm infection on host gene expression in the blood of SMs during the first 2 weeks of infection. More specifically, we observed that (a) the bulk of differential gene expression is detected in the blood at days 7, 10, and 14 after infection; (b) very little differential gene expression is detected at day 3 after infection; and (c) 
A

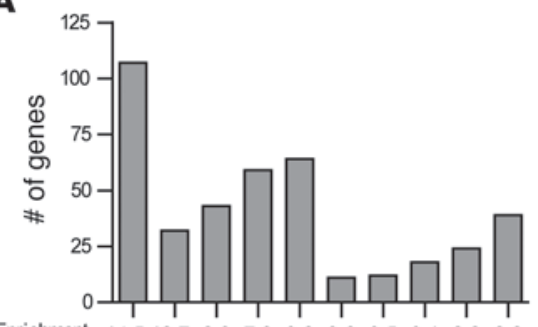

$\begin{array}{llllllllllll}\text { Enrichment } & 14.5 & 12.7 & 8.0 & 7.0 & 3.8 & 3.6 & 3.5 & 3.1 & 2.8 & 2.6\end{array}$ Score

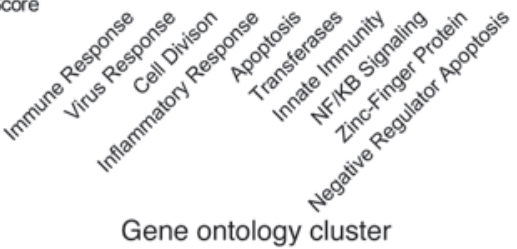

B

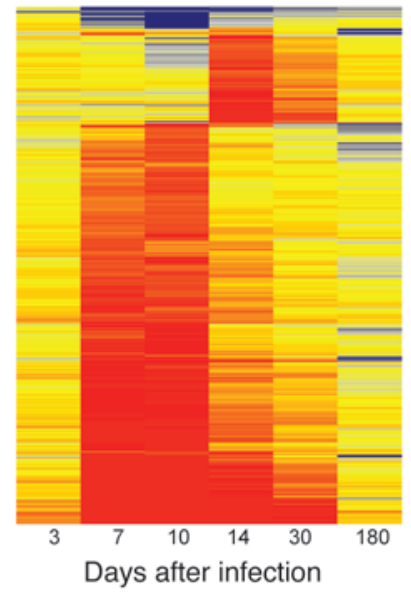

C

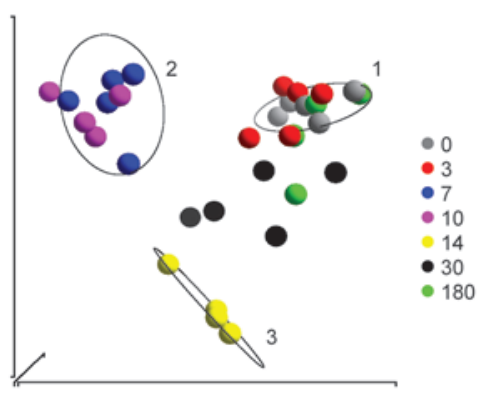

D

TLR5
PTX3

NBN

TIFA

TLR6
TREM1

TRIM25

TLR2

TRIM26

ZC3HAV1

TRAFD1

TRIM38

NAGK

CYBB

$E M B$

CD68

TRIM21

TDRD7

UNC93B1

CLEC7A

CD163

TRIM5

EIFAK2

CD164

TRIM22

RIN2

UBE2L6

PLSCR1

TNFAIP6

BST2

RNF213

CCDC75

TRIM14

HERC6

DDX58

USP18

HERC5

RSAD2

APOBEC $3 \mathrm{H}$

Innate Immunity
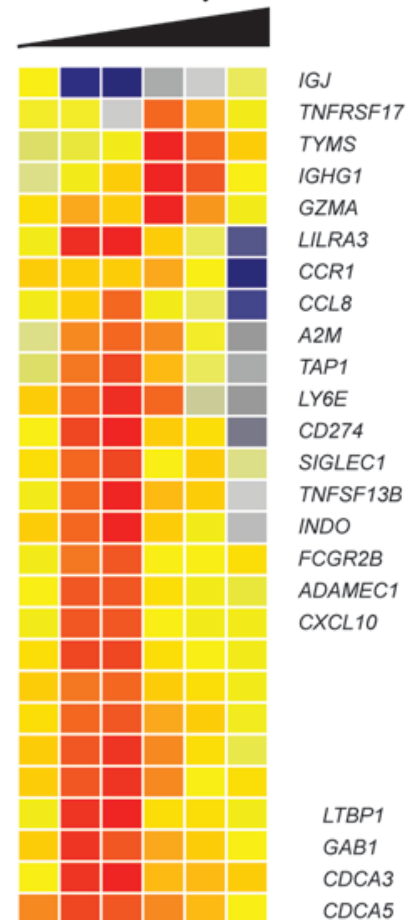

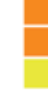

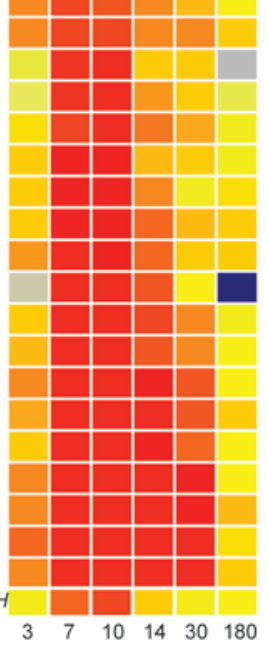

LMO2

PARP11

WARS

SP100

NM1

PARP12

$M A F B$
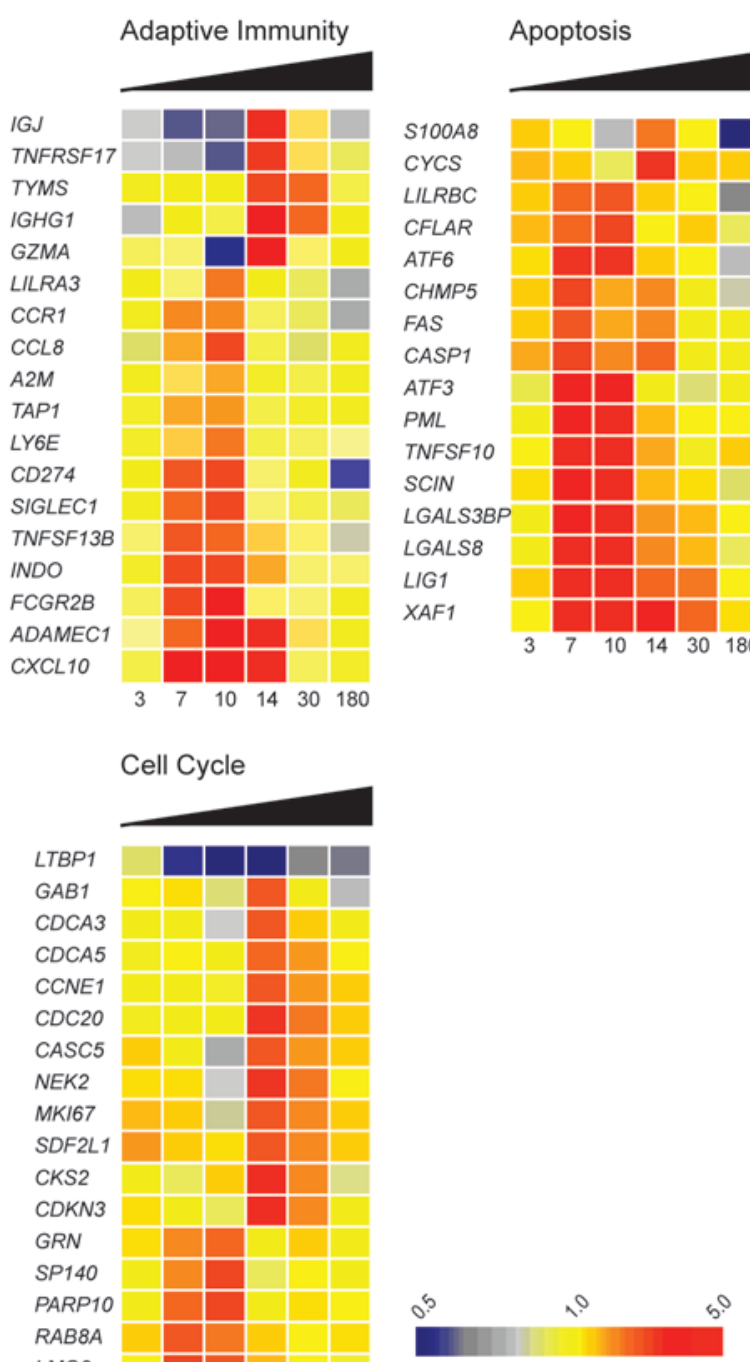

DTX1

PARP9

SECTM1
Linear fold change

Figure 3

Gene-expression profile of the immune response to SIV infection in SMs. (A) Ontology profiling of 428 probe sets significantly altered by SIV infection in SMs was based on gene ontology annotations retrieved from the DAVID Bioinformatics Database. Columns represent the number of genes in each ontology cluster listed below the $x$ axis. Enrichment scores were calculated using the human genome as a background. (B) Hierarchical clustering of 428 genes significantly regulated by SIV infection in SMs was performed as described in Figure 1 legend. Clustering was performed on the average of $\log _{10}$ ratios of gene expression relative to levels before infection; fold changes were calculated by subtracting the $\log _{10}$ intensity preinfected measurement from after-infection measurement for individual animals prior to calculating the average. The dendrogram is not shown. (C) PCA of $\log _{10}$ intensity measurements of 428 differentially expressed probe sets. Colored circles represent data set for individual SMs at the time points indicated in the key. Ellipses are centered on the median of the following time points: days 0 , 10, and 14. (D) Heat maps of selected innate immunity, adaptive immunity, apoptosis, and cell-cycle regulating genes with differential expression in SIV-infected SMs. Genes were categorized based on annotation in DAVID, Entrez Gene (http://www.ncbi.nlm.nih.gov/sites/entrez?db=gene), and Ingenuity Pathway Assist databases and on described function in the literature. Genes covered by multiple probe sets on the array are represented by the highest intensity. Genes were clustered as described in Figure 1 legend. Color scale is shown at bottom and ranges from 2-fold downregulated to 5-fold upregulated. 
A

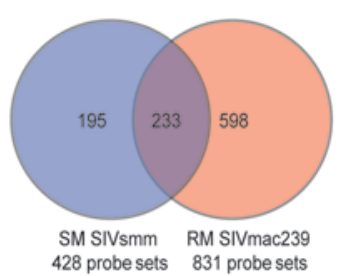

C
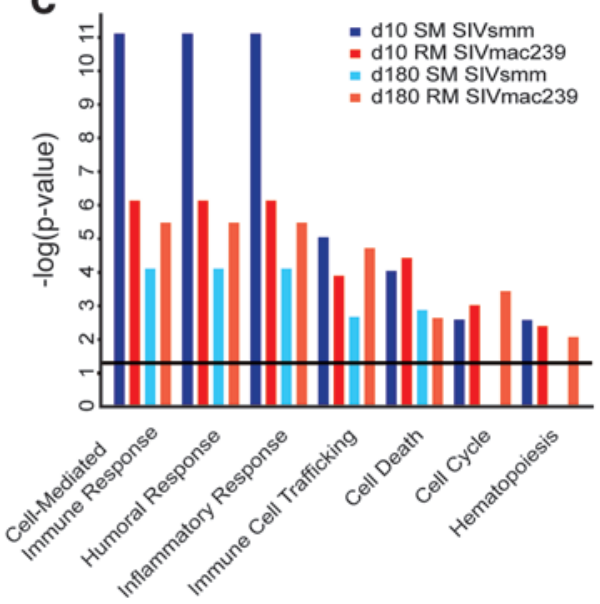

B RM SIVmac239

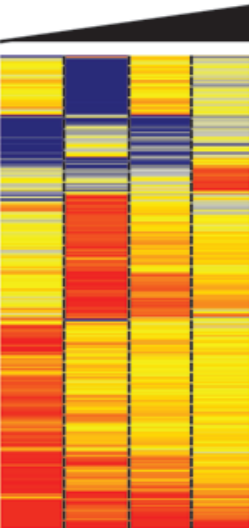

$10 \quad 14 \quad 30 \quad$ Chr

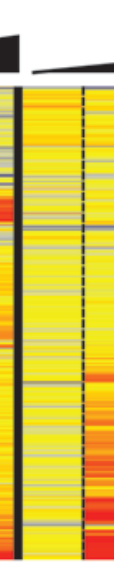

RM SIV smm
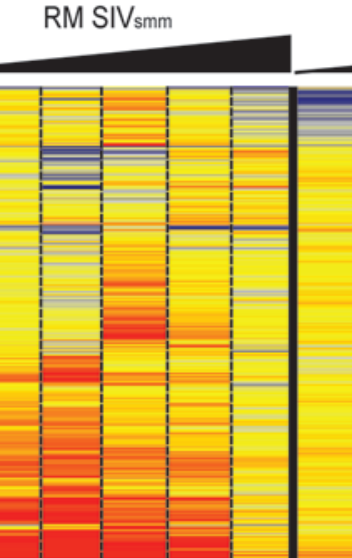

SM SIV smm Days after infection

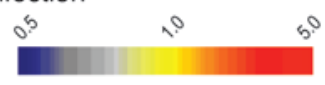

Linear fold change

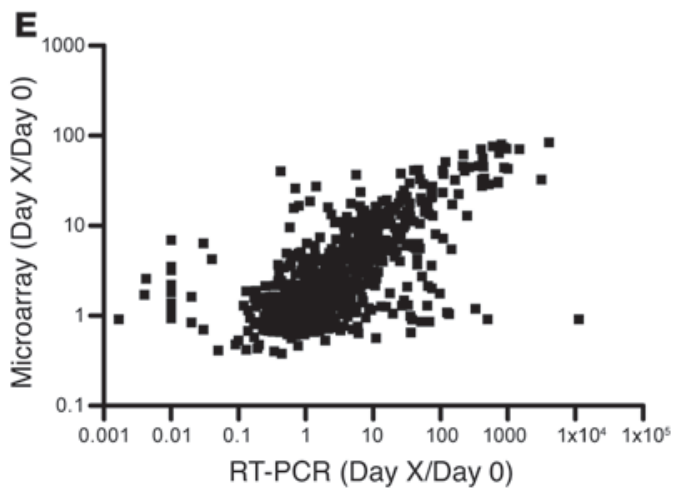

D
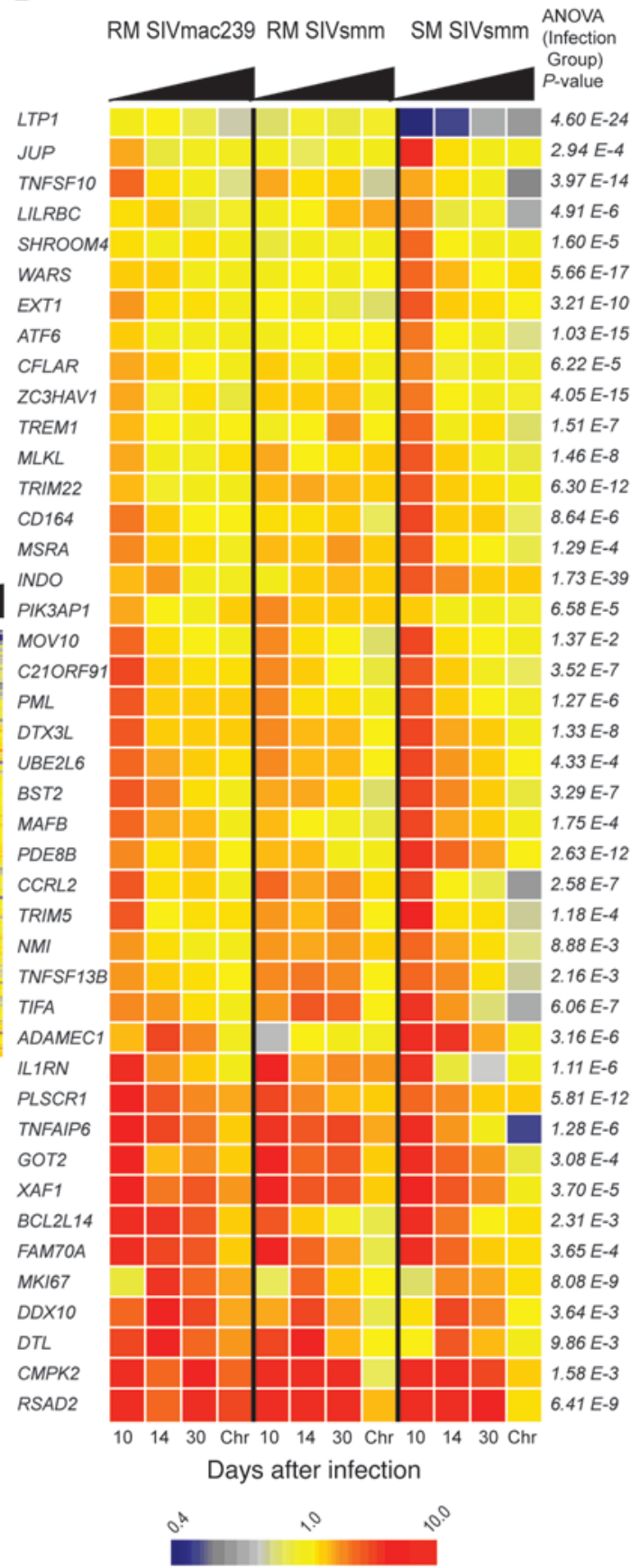

Linear fold change 


\section{Figure 4}

SMs and RMs exhibit distinct molecular signatures upon SIV infection. (A) Venn diagram indicating overlap of /probe sets with differential expression in SMs and RMs in response to SIV infection. (B) Heat map of 1025 probe sets induced in SIVsmm-infected SMs and/or SIVmac239-infected RMs. Chr, chronic. (C) Ontology enrichment comparison of SIV-inducible genes was performed using Ingenuity Pathway Analysis. Genes with significant differential expression at day 10 and day 180 in SIVsmm-infected SMs and SIVmac239-infected RMs (indicated by colored bars) were analyzed for annotation against all genes for a given function in the Ingenuity database using right-tailed Fisher's exact test. A significance threshold of $P=0.05$ is indicated by the horizontal line. (D) Heat map of 43 probe sets significantly induced by SIVsmm infection in SMs and judged significantly different between infection groups. The heat maps depict probe sets with the largest magnitude of gene-expression fold change and/or known function in immune responses, apoptosis, or cell cycle. For $\mathbf{B}$ and $\mathbf{D}$, genes were organized in heat maps using hierarchical clustering as described in Figure 1 legend and in Supplemental Methods. "Chronic" refers to day 180 in the SIVsmm-infected SMs and RMs and day 184-224 in SIVmac239infected RMs. Relative gene-expression changes are depicted by the color scales below heat maps. (E) Correlation between relative fold changes measured by microarray and qPCR. Each point represents the fold change of a single gene relative to day 0 for individual animals at a given time point; $x$ and $y$ axes are microarray and qPCR $\log _{10}$ fold changes, respectively. Number of $X Y$ pairs $=969$. Pearson's $r$ correlation $=0.6614 ; 95 \% \mathrm{Cl}=0.6244$ to $0.6954 ; P<0.0001$ (2-tailed).

the majority of the upregulated genes return to baseline levels of transcription by day 180 after infection (Figure 3B). Visual reduction of the data set using PCA demonstrated a similar pattern, as the profiles of gene expression for individual animals in samples collected prior to SIV infection and in those collected at days 3 and 180 after infection were indistinguishable (Figure 3C). The profiles of gene expression at days 7,10, and 14 after infection were highly dissimilar from each other and from baseline, while the transcript profiles at day 30 were intermediate, indicating that the wave of gene induction that occurs during acute SIV infection has begun to subside by day 30 after infection (Figure 3C). Only 6 probe sets were detected as having differential expression at day 180 after infection; interestingly, 3 of these gene products (LY96/MD2, TNFAIP1, and S100A8) are putative mediators of inflammation and were all significantly downregulated (Figure 3D). Collectively, these data indicate that SIVsmm infection of SMs is far from being transcriptionally silent and that dramatic changes in the profile of gene expression occur during the acute stage of infection (i.e., up to day 30). Interestingly, despite the induction of more than 400 genes during primary SIV infection of SMs and the maintenance of plasma viral loads of $10^{5}$ copies $/ \mathrm{ml}$ throughout the follow-up, only a handful of genes were found to be different from baseline during chronic infection.

Early induction of genes regulating innate immunity in SIV-infected SMs. To organize the list of genes induced by SIV infection in SMs into categories related by ontology, we utilized the DAVID Bioinformatics Resources database (http://david.abcc.ncifcrf. gov) (33), which clusters genes with related gene ontology terms into functional groups. As shown in Figure 3A, genes with functions associated with immune responses, cell division, apoptosis, and inflammation were enriched in the data set of 428 genes induced after SIV infection. Using this provisional classification, we focused on genes related to innate immunity, adaptive immunity, cell cycle, and apoptosis (Figure 3D). This ontology analysis revealed that a strikingly large number of molecules regulating innate immunity were differentially expressed in SIV-infected SMs. These data are summarized in the heat map in Figure 3D and in Supplemental Table 4. The most remarkable finding was that a large number of genes identified as ISGs were upregulated during acute SIVsmm infection of SMs (see below for more details). SIVsmm infection of SMs also induced the upregulation of several pattern-recognition receptors (PRRs), including TLR2, TLR5, TLR6, and LY96/MD2, at days 7-10 after infection. Similarly, we observed upregulation of the probe sets for DDX58/RIGI, a non-TLR PRR that initiates type I IFN responses through IFN regulatory factor 3 (IRF3) and IRF7 $(34,35)$ during the acute phase of SIV infection (Figure 3D). The analysis of genes clustered within the innate immunity functional group also revealed that primary SIVsmm infection of SMs is associated with marked upregulation of a number of known viral restriction factors, including TRIM5A, TRIM22, TRIM25, PML/ TRIM19, APOBEC3H, and BST2/tetherin (where BST2 indicates bone marrow stromal antigen-2.) (Figure 3D).

Collectively, these data indicate that acute SIVsmm infection of SMs is associated with a rapid and dramatic upregulation of numerous genes involved in the generation of innate antiviral immune responses, including ISGs and cell factors that restrict retrovirus replication.

Induction of genes involved in the generation and regulation of adaptive immunity in SIVsmm-infected SMs. We next focused our analysis on the impact of SIVsmm infection of SMs on the transcriptional profile of genes involved in the generation of an adaptive immune response to the virus. In this regard, we observed (a) upregulation of the CTL-related gene encoding granzyme A (GZMA) at day 14 after infection; (b) upregulation of the chemokine receptor CCR1, which is expressed on activated T cells, at days 7 and 10 after infection; (c) upregulation of 2 chemokines regulating migration of activated effector T cells, CCL8/MCP2 (a CCR1 and CCR2b ligand) and CXCL10/IP10 (a CXCR3 ligand), at days 7 and 10 after infection; (d) increased expression of the B cell inhibitory receptor TNFRSF17/CD268/BAFFR and its ligand, TNFSF13B/CD257/ $B A F F$, at days 14 and 30 after infection and days 7,10 , and 14 after infection, respectively; (f) induction of the gene ADAMDEC1, whose product is thought to mediate the interaction of DCs and germinal center T cells in the LN at day 30 after infection; and (g) upregulation of the immunoglobulin genes IGHG1/IGG1 and IGJ at days 14 and 30 after infection.

Perhaps more interestingly, we found that acute SIVsmm infection of SMs is associated with upregulation of several genes that are involved in the active modulation of an ongoing innate and adaptive immune response (Figure 3D). These genes included (a) the immune regulatory molecule $C D 274 / P D L 1$, which suppresses T cell activation via binding of PD1 (36) (upregulated at days 7 and 10 after infection); (b) the immune regulatory enzyme IDO1/INDO, which inhibits $\mathrm{T}$ cell proliferation via tryptophan metabolism (37) (induced at day 7 after infection and maintained throughout the chronic stage of infection); (c) LILRA3/ILT6, a member of the leukocyte immunoglobulin receptor (LIR) gene family (transiently upregulated at day 10 after infection); and (d) the LILRBC gene, an ortholog of the inhibitory, type B family of human LIRs (upregulated at days 7 and 10 after infection).

Collectively, our observation of early upregulation of selected adaptive immune effector genes as well as genes involved in immune modulatory pathways suggests that SIV-infected SMs 

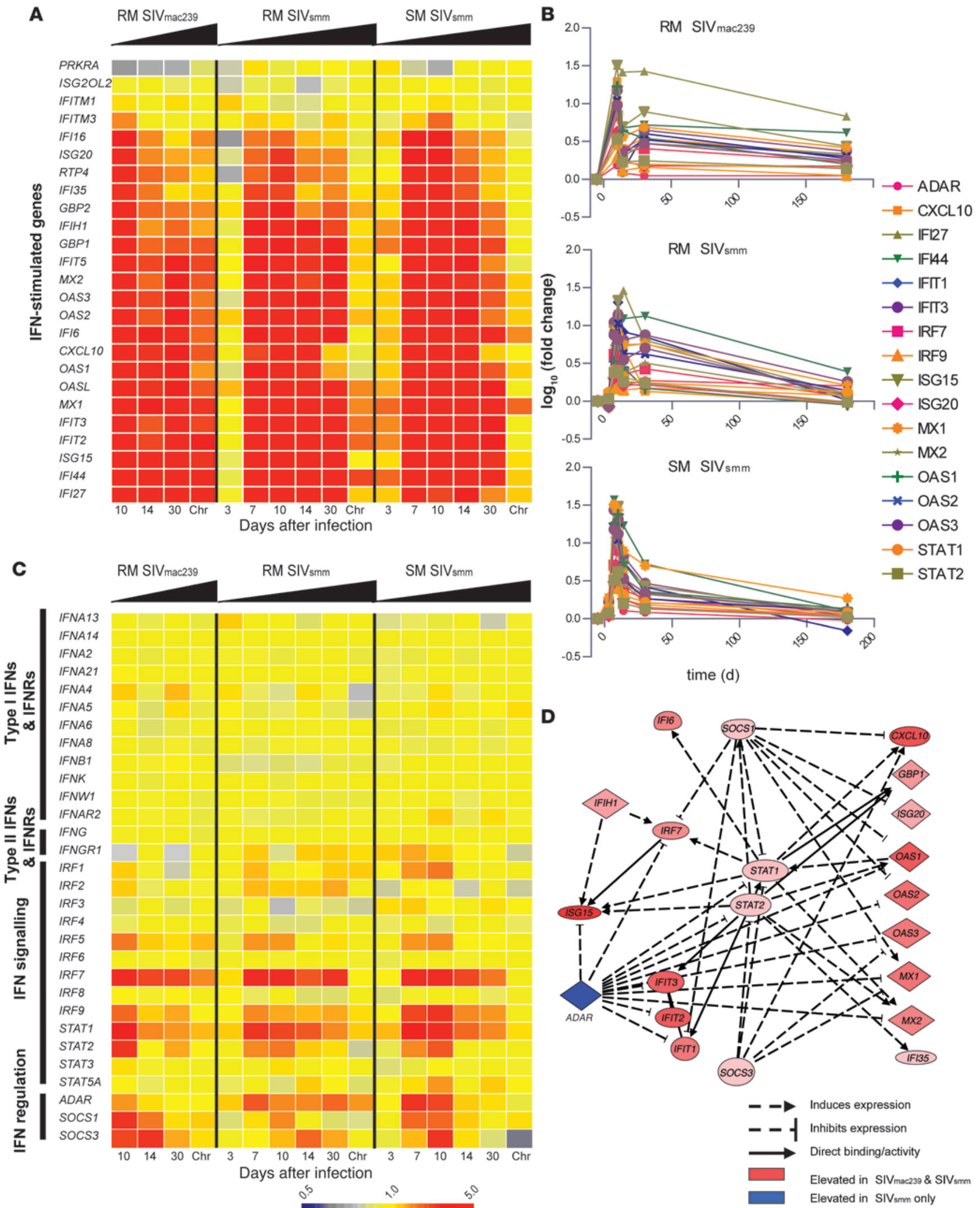

Linear fold change 


\section{Figure 5}

SIV infection in SMs induces extensive ISG expression during acute infection. (A) Heat map of ISGs expressed during SIV infection in SMs and RMs. Genes were organized using hierarchical clustering as described in Supplemental Methods. ISG classification was limited to canonical ISGs and/or those with well-established IFN induction. (B) Longitudinal analysis of expression for 25 ISGs measured by microarray. (C) Heat map of type I and II IFNs and IFN pathway-regulating molecules. For $\mathbf{A}$ and $\mathbf{C}$, the scale is indicated at bottom. (D) Network analysis of ISG expression was performed using Ingenuity Pathway Analysis Network Generation tool. Genes significantly regulated at day 10 in SIVsmm-infected SMs and SIVmac239-infected RMs were analyzed, and the top-scoring network was used as a starting reference and updated according to the most recent literature.

mount an early adaptive immune response to the virus in which rapid induction of immune regulatory mechanisms likely results in limited immune activation during chronic infection.

Expression of cell-cycle proteins during acute SIVsmm infection of SMs. Acute SIVsmm infection of SMs is associated with significant but transient increase in the expression of the cell-cycle-related marker Ki-67 on T cells, consistent with a state of active T cell activation and proliferation (18). To further investigate the nature of this phenomenon, we focused our attention on the expression of genes related to cell cycle in our cohort of SIVsmminfected SMs. We found that cell-cycle genes with transcriptional upregulation fell into 2 patterns of expression, i.e., those induced at day 7 after infection and those induced at day 14 after infection (Figure 3D). The genes induced at day 7 included 4 members of the poly (ADP-ribose) polymerase (PARP) family: PARP9/BAL1, PARP10, PARP11, and PARP14/BAL2, indicating possible coregulation of these proteins. Proteins of the PARP family modify histones and other nuclear proteins in response to DNA damage; their cleavage by caspases induces apoptosis in cells of both lymphoid and nonlymphoid lineage $(38,39)$. This rapid upregulation of PARP family members in response to SIV infection is consistent with the initiation of a coordinated program of gene expression aimed at limiting cell death during the priming of antiviral innate and adaptive immune responses. Cell-cycle genes that were induced at day 14 after infection included classical markers of proliferation (CCNE1, CDCA3, CDCA5, CDC20, and MKI67). Taken together, these data indicate the presence of active, albeit transient, upregulation of the expression of many cell-cycle-related genes during acute SIVsmm infection of SMs.

Induction of apoptosis genes during acute SIVsmm infection of SMs. To further investigate the kinetics of immune activation during acute SIVsmm infection of SMs, we next screened our list of genes induced by the infection for those with function related to apoptosis and/or activation-induced cell death (Figure 3D). Previous studies have shown that SIV infection of SMs is associated with increased $\mathrm{T}$ cell apoptosis during acute but not chronic infection $(28,31)$. Here we found that acute SIVsmm infection of SMs is associated with rapid induction of the apoptosis-related genes TRAIL, FAS, and CASP1, which peaked at day 7 after infection and returned to preinfection levels by day 30. It is interesting to note that CFLAR/cFLIP, which inhibits the extrinsic pathway of apoptosis in T lymphocytes (40), was also overexpressed during this interval. Our data indicate that apoptotic processes are transiently induced in the acute phase of SIVsmm infection in SMs and suggest that the concomitant upregulation of antiapoptotic molecules reflects the resolution of this early apoptosis induced by SIV infection.

Comparison of SIV-induced gene expression in SMs versus RMs. The observation that SIV infection is nonprogressive in SMs despite uncontrolled virus replication suggests that SIV/HIV-induced pathogenesis is not simply a consequence of the direct cytopathic effect of the virus but also involves species-specific features of the host response to the infection (15). Here, we hypothesized that comparing the host-expression profile of SMs with that of RMs would identify a molecular signature associated with SIV-induced pathogenesis or, alternatively, with resistance to disease. To this end, we infected 2 cohorts of RMs with either SIVsmm $(n=4)$ or SIVmac239 $(n=8)$, as described in Figure 1A. Based on the observed viral load and CD4 kinetics (Figure 1, B and C), we focused on the blood samples of our cohort of SIVmac239-infected RMs as a representative model of pathogenic infection to compare with SMs. We identified genes induced in SIVmac239 infection of RMs using the same algorithm employed in our analysis of SMs, with differential genes defined as follows: (a) a significant variance in their $\log _{10}$ intensity after infection $(P<0.00746)$; and (b) a 2 -fold or greater change in average expression relative to preinfected values in 1 or more time points. Despite these stringent parameters, we identified 830 probe sets with differential expression (Supplemental Table 5) after SIVmac239 infection (compared with 428 probe sets identified in SMs). Of these transcripts, 233 were common to both lists, 195 were specific to SIVsmm-infected SMs, and 597 specific to SIVmac239infected RMs (Figure 4A). Hierarchical clustering demonstrated that the bulk of differential gene expression in SIVmac239-infected RMs was concomitant to that of SIVsmm-infected SMs (i.e., occurring by day 10 after infection), with markedly reduced differential gene expression during the chronic phase (Figure 4B). Notably, we observed that numerous genes were upregulated in the chronic phase of SIVmac239 infection of RMs but not during chronic SIVsmm infection of SMs (Figure 4B). Using ontology profiling, we compared the lists of differentially expressed genes from day 10 and day 180 after infection in SIVmac239-infected RMs and SIVsmminfected SMs (Figure 4C). At day 10 after infection, the transcriptional profile in SMs revealed greater upregulation of genes associated with both cellular and humoral immune response when compared with SIVmac239-infected RMs, as determined by both the absolute number of genes and enrichment score (Figure 4C). However, by day 14 after infection, this trend was reversed, with a greater enrichment of cell-mediated immune response genes in SIVmac239-infected RMs, showing comparison with LN data). At day 180 after infection, gene expression associated with an adaptive immune response had returned to baseline in SMs, while 16 genes were still upregulated in SIVmac239-infected RMs (Figure 4C). Striking differences between SMs and RMs were observed in the chronic phase of SIV infection for genes associated with the cell cycle, indicating persistently high transcriptional activity in RMs but not in SMs. Collectively, these data demonstrate that SMs and RMs experience a robust innate and adaptive immune response during the acute phase of SIV infection, but these responses are almost completely abrogated during chronic infection in SMs. In contrast, in pathogenic SIVmac239 infection of RMs, gene expression indicative of immune activation is detectable well into the chronic phase of infection.

To then identify specific genes that are differentially expressed in the SIVsmm-infected SMs, SIVsmm-infected RMs, and SIVmac239-infected RMs, we employed a modified 1-way ANOVA 


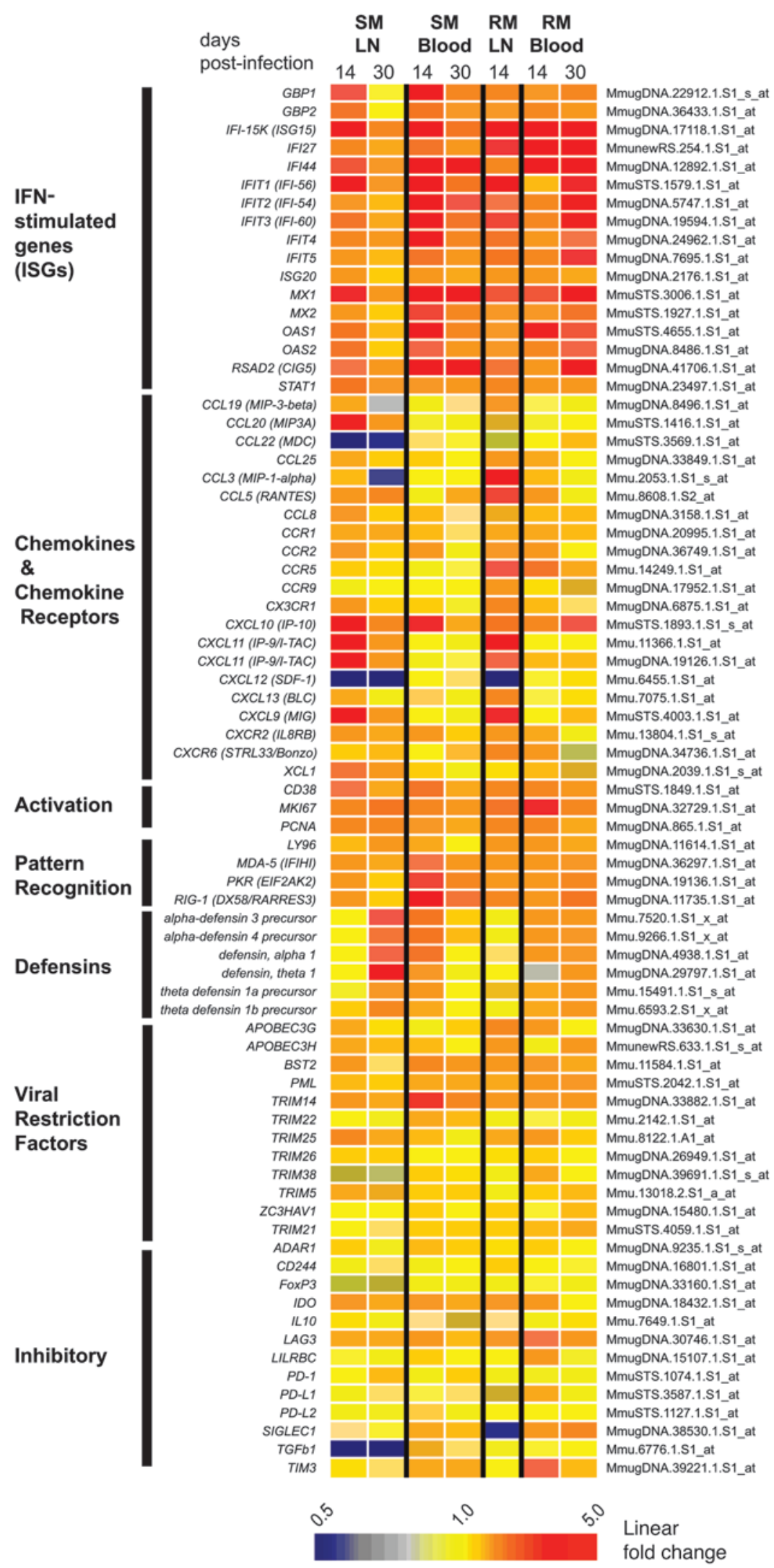

Figure 6

Gene-expression profiles in LNs of SIV-infected SMs and RMs. Heat map of genes induced in LNs and blood at 14 and 30 days after infection with SIVsmm or SIVmac239. Differentially expressed genes in the LNs were defined as those determined significant $(P<0.05)$ by 2 -sample Wilcoxon's signed-rank test and an average 2 -fold change relative to uninfected samples.

(detailed description in Supplemental Methods) that identified transcripts in blood that were (a) significantly different among the 3 cohorts and (b) significantly different between the SM cohort and both RM cohorts, as determined by pairwise comparison. The large number of genes with differential transcript levels between species is likely due to (a) species-specific differences in constitutive gene expression unrelated to SIV infection; (b) differential induction following SIV infection; and (c) possible interspecies differences in probe set hybridization affinities. To gain insight into the molecular signature associated with SIV-induced pathogenesis (or lack thereof), we limited our analysis to those genes with SIV-inducible expression in SMs and filtered our gene list to 239 candidates (Supplemental Table 6). We then further narrowed this list to 45 genes based on the magnitude of expression differences or involvement in immune processes, cell cycle, or apoptosis based on ontology profiling (Figure 4D). More specifically, we found that SIVsmm-infected SMs exhibit (a) lower expression of several proapoptotic genes, including TNFSF10/TRAIL, BCL2L14, and XAF1, as well as increased expression of the antiapoptotic regulator CFLAR/cFLIP during the acute phase of SIV infection; (b) increased expression of the host-restriction genes TRIM22 and ZC3HAV1/ZAP during the acute phase and lower expression of PML/TRIM19 and RSAD2/Cig5/Viperin during the chronic phase; (c) decreased expression of MKI67 during chronic infection; (d) increased levels of 2 genes involved in tryptophan metabolism, i.e., WARS/IFI53, an IFN-induced tryptophanyl-tRNA synthetase that binds to HIV Gag (41), and IDO1/ INDO. Of note, BST2/tetherin, another important host-restriction factor $(42,43)$, exhibited strong induction in both SIVsmm-infected SMs and SIVmac239-infected RMs but not in SIVsmm-infected RMs. Real-time PCR quantitation (qPCR) of 14 genes demonstrated high concordance $(r=0.6614$, $P<0.0001, n=969$ ) with the array data (Figure 4E and Supplemental Figure 2).

SIV infection of SMs is characterized by robust but transient upregulation of IFN-stimulated gene expression. Type I IFNs are produced in response to viral infections and initiate signal transduction cascades resulting in the transcription of many downstream antiviral effector molecules, collectively termed ISGs (44). To focus on the expres- 
A

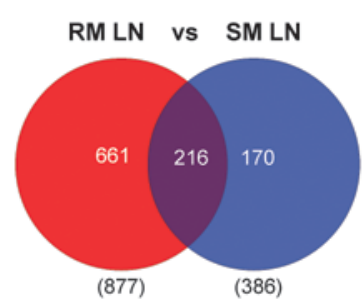

SM LN vs SM blood
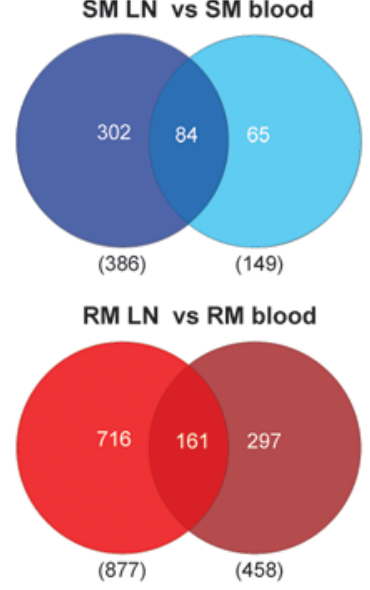

B

Cell Cycle

Cell-mediated

Immune

Response

Cellular

Movement

Cell Death

Antigen

Presentation

Immune Cell

Trafficking

Cell-Cell

Interaction

Hematological

System

Development

Hematopoiesis

Humoral

Immune

Response

Lymphoid Tissue

Structure \&

Development

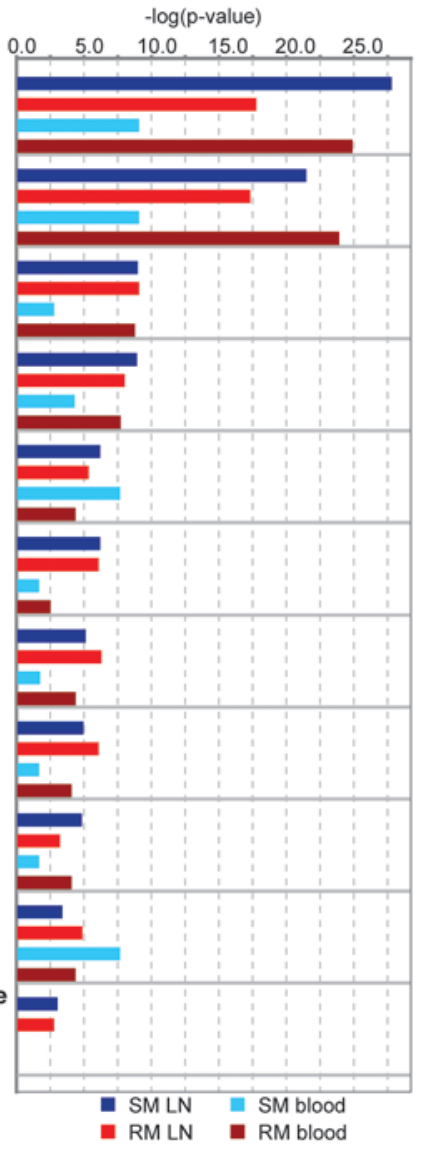

C

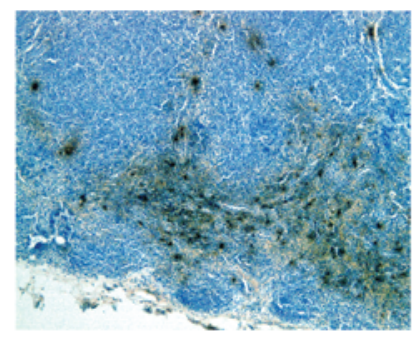

\section{Figure 7}

Comparative analysis of gene expression profile in response to SIV infection in lymph nodes and peripheral blood of SMs and RMs. (A) Venn diagrams of Boolean relationships between sets of differentially expressed genes at day 14 after infection; the comparison groups are indicated at top, with the values in parentheses indicating the total number of differential probe sets in the respective data set; values within Venn diagrams indicate differential probe sets. (B) Functional pathway analysis was performed using the Ingenuity database as described in the Figure 4 legend. (C) Collections of dark staining $\alpha$-defensin ${ }^{+}$cells in blue-counterstained LN section from a representative SM section at day 30 after infection. Original magnification, $\times 20$.

sion of IFNs and regulated genes, we filtered our data set to include a set of 25 well-characterized ISGs as well as type I and II IFNs and their receptors, downstream signal transduction molecules, and regulatory proteins. As shown in Figure 5, A and B, robust expression of at least 21 individual ISGs in all 3 groups of SIV-infected animals was observed during acute infection. Interestingly, the highest $\log _{10}$ ratios of ISGs were observed at day 10 after infection in the SMs compared with SIVsmm- or SIVmac239-infected RMs, indicating an intense type I IFN response in SIVsmm-infected SMs. ISG expression in the chronic phase was vastly different between RMs and SMs, with the latter downmodulating 20 out of 22 ISGs to baseline levels despite maintaining an average plasma viral load of approximately $3.7 \times 10^{4}$ copies $/ \mathrm{ml}$ in chronic infection (Figure 1B). In contrast, SIVsmm-infected RMs showed chronic overexpression of 6 ISGs, and SIVmac239-infected RMs maintained significant upregulation of 20 ISGs that were induced during acute infection (Figure 5, A and B). It is notable that during chronic infection, SIVsmm-infected RMs maintained ISG levels higher than those in SIVsmm-infected SMs, although their average plasma viremia was more than 2 log lower (Figure 1B). Taken together, these data clearly indicate a strong, albeit largely transient, induction of IFN-stimulated gene expression in SMs during acute SIV infection. qPCR validation of 10 ISGs across the 3 groups of animal confirmed the findings of the microarray analysis (Supplemental Figure 2).

We next categorized all available probe sets representing type I and II IFNs and their receptors, genes from closely related signaling pathways, and known regulators (Figure 5C). Our analysis did not reveal any significant upregulation of any type I IFNs, type II IFNs, or their receptors in SIV-infected SMs or RMs (Figure 5C), and similarly, qPCR did not detect appreciable levels of IFN- $\alpha$ or $-\beta$ (data not shown). In contrast, we observed significant induction of IRF7, IRF9, STAT1, and STAT2 expression in all 3 groups of SIV-infected animals. Similar to what we observed for ISGs, the upregulation of IRF7 was confined to the acute phase of SIV infection in SMs but persisted during chronic infection in SIVmac239-infected RMs. To confirm the ability of SMs to respond to IFN- $\alpha$, we stimulated PBMCs from 4 uninfected SMs with recombinant IFN- $\alpha$ and observed strong induction of ISG mRNA (Supplemental Figure $3 \mathrm{~A})$. As recent data have suggested that the type I IFN response can be desensitized in chronic HIV infection (45), we next determined whether SMs may become refractory to type I IFN stimulation dur- 

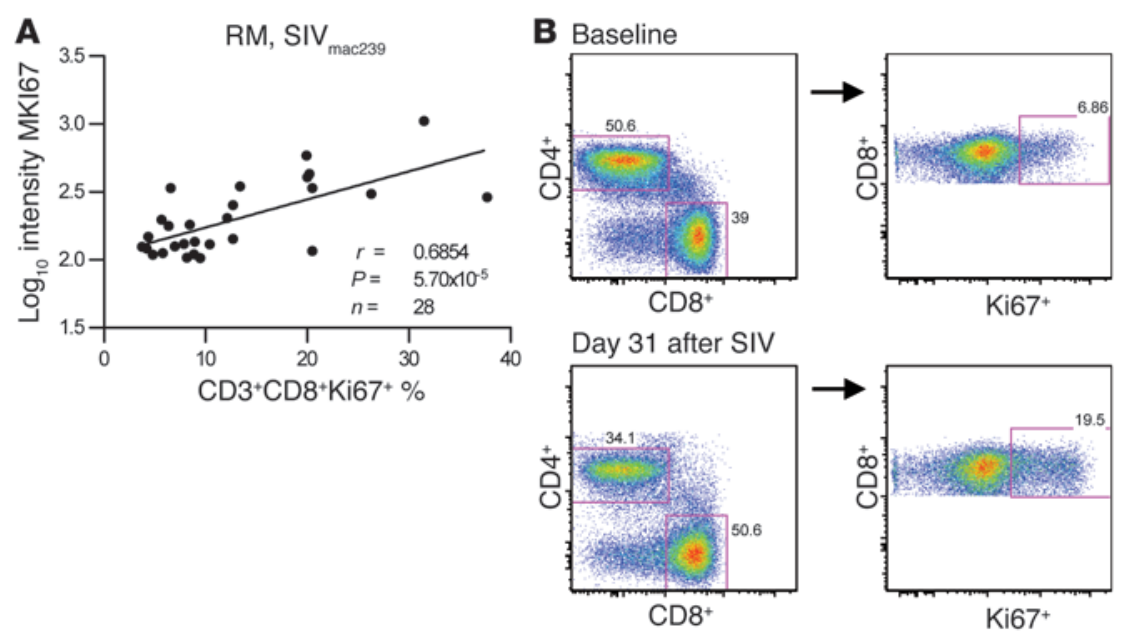
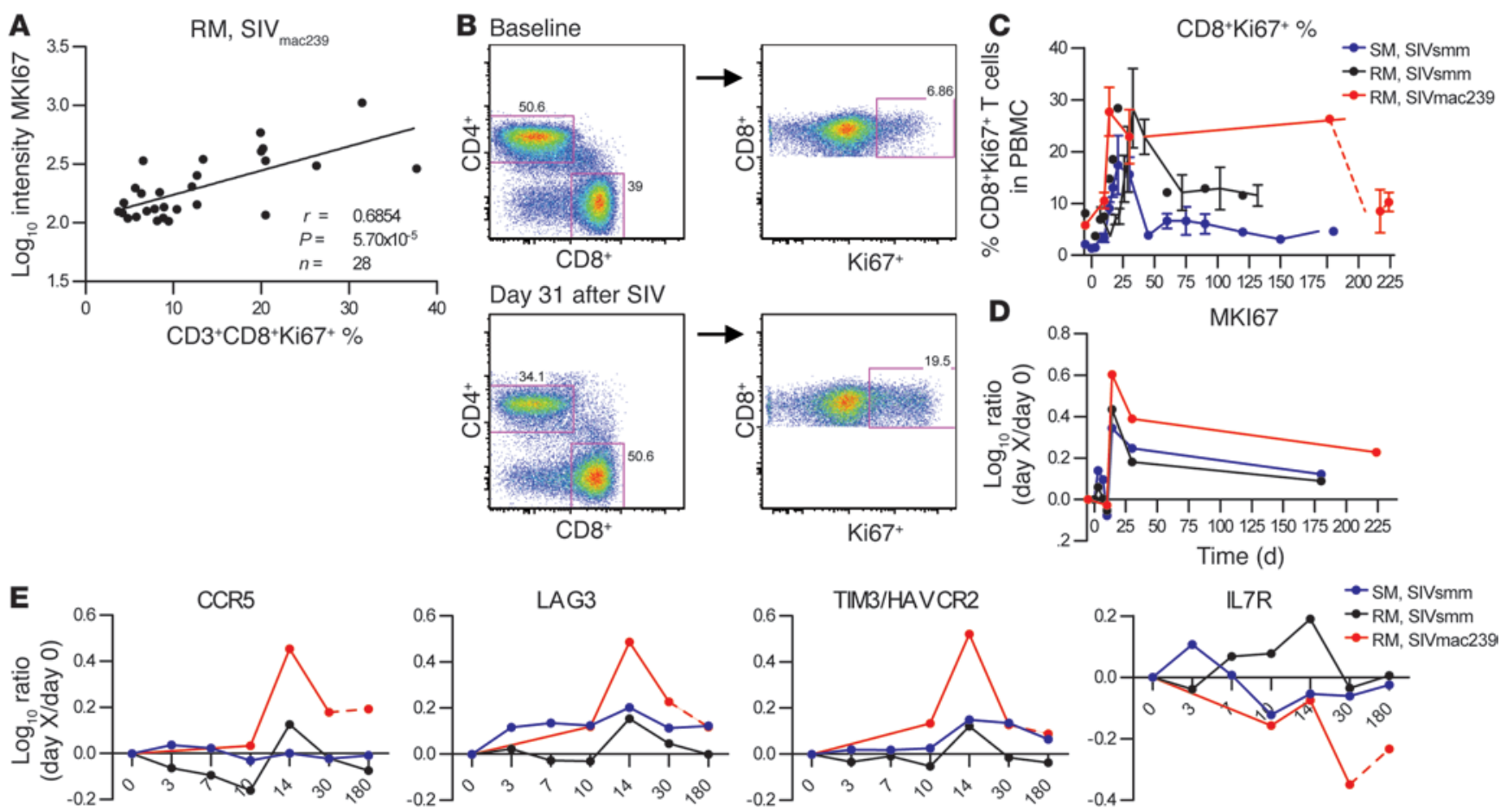

Days after infection

$\mathbf{F}$
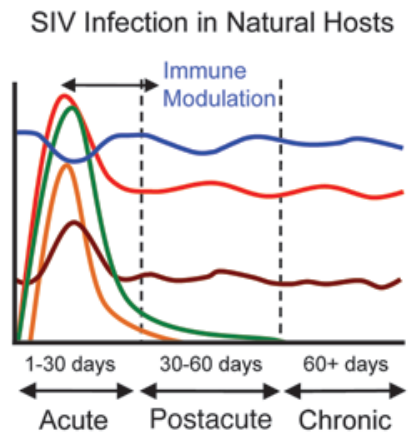

Pathogenic SIV Infection

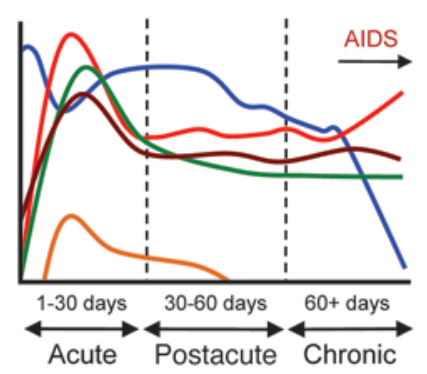

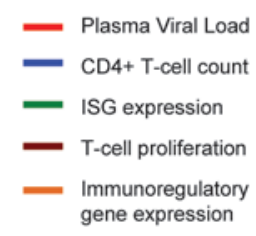

\section{Figure 8}

Systems biology identification of genes associated with immune activation in SIVmac239-infected RMs. (A) Pearson's correlation of CD8+Ki-67+ fraction with MKI67 gene expression in SIVmac239-infected RMs. Peripheral blood Ki- $67^{+} \mathrm{CD} 8 \%$ was assessed using the gating strategy outlined in B and was correlated with MKI67 mRNA $\log _{10}$ intensity in peripheral blood using Pearson's correlation (false discovery rate corrected; $P<0.00106$ ). (B) Representative density plot of flow cytometry analysis for expression of $\mathrm{Ki}^{-67^{+}}$on gated $\mathrm{CD} 3^{+} \mathrm{CD} 8^{+} \mathrm{T}$ cells in PBMCs of SIVmac239-infected RMs. The numbers denote the percentage of gated cells, indicating the fraction of CD $3^{+} \mathrm{T}$ cells expressing CD4 or CD8 (left panels) or the fraction of $\mathrm{CD}^{+}{ }^{+} \mathrm{CD} 8^{+} \mathrm{T}$ cells expressing Ki-67 (right panels) from a time point prior to SIV infection and from day 31 after infection in the same animal. (C) Longitudinal expression profile of $\mathrm{Ki}-67$ protein on $\mathrm{CD} 3^{+} \mathrm{CD} 8^{+} \mathrm{T}$ cells as measured by flow cytometry; error bars indicate SEM. (D) MIK67 mRNA as measured by microarray. MKI67 gene expression is the average ratio of gene expression relative to preinfected samples. (E) Longitudinal profiles of 4 genes whose expression significantly correlated with $\mathrm{CD} 8^{+} \mathrm{Ki}-67^{+}$percentage in peripheral blood and identified in the literature as related to immune activation, CD8 ${ }^{+}$T cell exhaustion, or HIV pathogenesis. (F) Model of immunomodulation during SIV infection of natural hosts and during pathogenic infection, demonstrating differences in ISG induction and immunoregulatory gene expression; details in text.

ing chronic SIV infection. To this end, we isolated PBMCs from 4 of the SIVsmm-infected SMs used for the array analysis. Although the SMs were chronically infected for over 3 years, IFN- $\alpha$ stimulation of PBMCs induced ISG expression at a level comparable to that of uninfected animals (Supplemental Figure 3B).

The diminished type I IFN response observed in the postacute phase of SIV infection in all 3 groups of animals could reflect the coincident decrease in viral load. However, the fact that only SIVsmm-infected SMs experience a near-complete resolution of ISG expression in the chronic phase of infection despite a post- peak decline in viremia that is intermediate between those of SIVmac239- and SIVsmm-infected RMs suggests the presence of active immune regulatory mechanism in these animals. We examined the expression of known negative regulators of IFN signaling and observed that gene expression of SOCS1 and SOCS3 was relatively similar in SIVmac239-infected RMs and SIVsmm-infected SMs (Figure 5C). In contrast, expression of the putative IFN- and ISG-suppressing gene adenosine de-aminase RNA-specific (ADAR) was persistently elevated in SMs but not in SIVmac239-infected RMs (Figure 5C). Network analysis of the expression of ISG- and 
IFN-regulating genes in SIVsmm-infected SMs and SIVmac239infected RMs demonstrated the central regulatory roles of $A D A R$, SOCS1, and SOCS3 in IFN signaling (Figure 5D).

Collectively, these data demonstrate that both SIVsmm infection of SMs and SIVmac239 infection of RMs are associated with early and massive upregulation of ISG expression. However, only RMs maintained this phenotype during chronic infection.

Profile of gene expression in the LNs of SIV-infected SMs and RMs. Many studies have shown that LNs are key anatomic compartments in the interplay between HIV and SIV and the primate immune system, acting as both reservoirs of virus production and sites of immunopathology during chronic SIV and HIV infections (46-48). To investigate how SIV infection has an impact on the transcriptional profile of LNs in our comparative model of RM and SM infection, we next assessed longitudinally the gene-expression profiles in LNs isolated from a subset of animals included in our study (see Supplemental Methods) and compared them with those observed in peripheral blood (Figures 6 and 7). We observed that, at day 14 after infection, the number of genes with differential expression in LNs upon SIV infection was nearly 3-fold higher than that determined in blood for both SMs and RMs (Figure 7A). Similar to what we found in blood, a greater number of genes were upregulated in RM LNs compared with SM LNs after SIV infection (Figure 7A). Ontology analysis of the expression profiles at day 14 after infection across tissues revealed that genes involved in cell cycle were the most highly enriched functional group in LN (Figure 7B). As expected, several genes regulating lymphoid-tissue development were present in the LN expression profiles but were absent from the blood data sets. Similar to what we found in blood at day 14 after infection, we observed that genes associated with cell-mediated immunity were more highly enriched upon SIV infection in the RM LNs compared with SMs (Figure 7B).

Detailed inspection of the lists of genes that are differentially expressed upon SIV infection in LNs of SMs and RMs (Supplemental Tables 7 and 8) demonstrated several key similarities with the genes that are differentially expressed in blood. In particular, we observed a strong induction of ISGs in the LNs of both SMs and RMs at day 14 after infection and a rapid decrease of ISG expression in the LNs of SMs by day 30 after infection (Figure 6). Consistent with our findings in blood, no elevations of mRNA for type I IFN was detected for either SM or RM LNs at any time point after infection (Supplemental Figure 4). It should be noted that the combined observations of broad ISG induction in both LNs and blood of SMs during acute SIV infection (Figure 5) provide a clear cross-tissue validation of the presence of an active type I IFN response during nonpathogenic infection of a natural host species.

While SMs and RMs showed a remarkable concordance in both blood and LN in the changes of ISG expression induced by SIV infection, we observed a marked species-specific divergence in the pattern of expression of chemokines and chemokine receptors (Figure 6). In particular, expression of CCL3 and CCL5 was markedly induced in RMs but not in SMs and, in agreement with our observations in the blood, the HIV/SIV coreceptor CCR5 was strongly induced during acute SIV infection in the LN of SIV-infected RMs but not SMs (Figures 6, 7, and 8). Of note, expression of the homeostatic chemokine CXCL12 was significantly reduced after infection in both species (Figures 6 and 7), perhaps suggestive of a switch in the LN transcriptome toward an inflammatory response.
In an attempt to identify potential mechanisms involved in the early resolution of immune activation in SIV-infected SMs, we examined the tissue-specific expression of several inhibitory cytokines and receptors. We found that a number of well-characterized immunoregulatory molecules (IL10, FOXP3, CD244) were not altered in either RM or SM (Figure 6). In addition, expression of $A D A R$, which was upregulated in the blood of SIV-infected SMs (Figure 5), was not observed in LNs of the same animals; however, strong induction of IDO was present in both the blood and LNs of SMs (Figure 6). Interestingly, expression of the inhibitory receptor lymphocyte activation gene 3 (LAG3), which was significantly increased (i.e., 3.1-fold) in the blood of SIV-infected RMs, was also increased in the LNs of the same animals to a level close to the 2-fold significance cutoff (i.e., 1.96-fold). In contrast, LAG3 expression in both LNs and blood of SIV-infected SM (1.4- and 1.6-fold, respectively) was well-below the cutoff and determined to be nonsignificant (Figure 6 and Figure 8D). While LAG3 upregulation in the LN of SIV-infected RMs was slightly below our 2-fold threshold for significance, the clear upregulation of this gene in the blood of these animals and also in the LNs of humans with acute HIV infection (49) makes LAG3 an intriguing candidate determinant of divergent immune regulation between pathogenic HIV/SIV infections of humans and RMs and nonpathogenic SIV infection of SMs.

In the last part of our comparative analysis in blood and LNs of SIVinfected SMs and RMs, we assessed the expression of several classes of antimicrobial and antiviral effector molecules in the 2 examined immune compartments. In contrast to blood, in which significant upregulation of several retroviral restriction factors (APOBEC $3 G$ and - H, TRIMs, BST2/tetherin, Z3CHAV1) was observed in both species at days 7 and 10 after infection (Figure 3D), significant upregulation in LNs was only observed for TRIM25, with the other restriction factors showing only modest and nonsignificant increases in gene expression (Figures 6 and 7). It should be noted that, since induction of restriction factors in blood peaked between day 7 and 10, earlier sampling may ultimately reveal a more robust expression of these factors in the LNs of SIV-infected RMs and SMs.

Finally, a striking contrast in expression patterns between SIVinfected SM and RM LNs was observed for genes encoding $\alpha$ and $\theta$ classes of defensins. Defensins are antimicrobial peptides that play a key role in the innate immune system of animals and plants (50). Defensin gene expression was observed at day 30 after infection in the LNs (but not blood) of SIV-infected SMs and was strongest for the $M$. mulatta $\theta$-defensin 1 gene (Figure 6). As shown in Figure $7 \mathrm{C}$, the increased expression of the $\alpha$-defensin 1 gene in the LNs of SIV-infected SM was confirmed by immunohistochemistry. In contrast, expression of defensin genes was low in both LNs and blood of SIV-infected RMs.

Molecular signature of immune activation in pathogenic SIVmac239 infection. Numerous studies indicate that HIV/SIV pathogenesis is associated with a state of chronic, generalized immune activation, which is characterized by increased protein expression of the activation markers HLA-DR, CD25, CD38, CD69, and Ki-67 (reviewed in ref. 51). In particular, Ki-67 has proven to be a reliable marker of SIV-associated immune activation in nonhuman primate studies (30). In this study, we sought to identify candidate genes that may influence the chronic immune activation associated with SIVmac239 infection of RMs by using a systems biology approach similar to that recently described by Querec and colleagues (52). To this end, we first assessed the level of immune activation in SIVmac239-infected RMs by measuring the protein 
expression of the activation markers HLA-DR, CD69, and Ki-67 on $\mathrm{CD}^{+}, \mathrm{CD}^{+} \mathrm{CD}^{+}$, and $\mathrm{CD}^{+} \mathrm{CD}^{+}$cell populations by flow cytometry and then examined the correlation of these protein levels with their corresponding gene expression. We found that the strongest correlation was between $\mathrm{Ki}-67$ on $\mathrm{CD}^{+} \mathrm{CD}^{+}$cells and gene expression of MKI67 in whole blood (Figure 8A and data not shown). Ki-67 protein expression in $\mathrm{CD}^{+} \mathrm{CD}^{+}$cells was transiently increased during acute SIVsmm infection of SMs but remained elevated throughout chronic infection in SIVsmm-infected RMs and, even more dramatically, in SIVmac239-infected RMs (Figure $8, \mathrm{~B}$ and C). As expected, changes in gene expression of MKI67 in whole blood measured by the array reflected those observed by flow cytometry (Figure 8D).

We then postulated that genes whose expression correlates strongly with the level of CD8 ${ }^{+} \mathrm{Ki}-67^{+} \mathrm{T}$ cells in the SIVmac239infected RMs may be candidate mediators of the immune activation observed during pathogenic SIV infection. Determination of the Pearson's correlation coefficient between the 830 probe sets differentially regulated by SIVmac239 infection in RMs and the level of $\mathrm{CD}^{+} \mathrm{Ki}-67^{+} \mathrm{T}$ cells identified 171 genes that were significantly correlated $(P<0.0106)$ (Supplemental Table 9). Several of those 171 genes have been associated with HIV pathogenesis, including (a) CCR5 ( $r=0.526, P=0.004)$; (b) IL7R $(r=-0.539, P=0.003)$; and (c) $\mathrm{T}$ cell immunoglobulin domain, mucin domain-3/hepatitis A virus cellular receptor 2 (TIM3/HAVCR2) $(r=0.572, P=0.0015)$ (Figure $8 \mathrm{E})$. Interestingly, we also noted that galectin-9, the TIM3 ligand, was upregulated at day 10 after infection in RMs infected with SIVmac239 but not in SIVsmm-infected SMs (Supplemental Table 5). In addition, we observed a highly significant, positive correlation between expression of LAG3 $(r=0.668, P=0.0001)$, a novel marker of T lymphocyte exhaustion (53), and the level of CD8 ${ }^{+} \mathrm{Ki}-67^{+} \mathrm{T}$ cells (Figure $8 \mathrm{E}$ ). These data indicate that this applied systems biology analysis is a viable approach to identifying candidate genes driving the pathogenic immune activation observed in HIV infection and implicate the lymphocyte-exhaustion markers TIM3 and LAG3 in the underlying molecular pathways.

\section{Discussion}

The mechanisms by which natural SIV hosts such as SMs and African green monkeys (AGMs) are able to remain disease free despite chronically high levels of virus replication remain poorly understood. In a series of studies, we and others have shown that a key feature of natural SIV infections is the absence of the chronic, generalized immune activation associated with pathogenic HIV and SIV infections of humans and RMs, respectively $(22,30,54-56)$. However, the causes of this difference in immune activation between natural and nonnatural hosts are still unclear. To elucidate potential molecular mechanisms responsible for the strikingly different outcomes of SIV infection between SMs and RMs, we conducted a comparative, longitudinal assessment of the profile of gene expression in 3 groups of SIV-infected nonhuman primates using high-density microarrays. We infected $5 \mathrm{SMs}$ with SIVsmm, 5 RMs with SIVsmm, and 8 RMs with SIVmac239 and measured gene expression at multiple time points during both acute and chronic infection. To the best of our knowledge, this is the first time that this type of analysis has been conducted in SIV-infected SMs, which represent the best-characterized model of natural SIV infection and are arguably the most relevant to human disease. Infection of RMs with the well-characterized SIVmac239 clone provided a comparison group for pathogenic infection, and
SIVsmm infection of RMs was included to control for gene-expression differences due to inoculum rather than host factors. The fact that none of the SIVsmm-infected RMs included in the current study experienced significant viremia or $\mathrm{CD} 4^{+} \mathrm{T}$ cell loss is not entirely surprising, since a previous study by our group showed that infection of RMs with primary, uncloned SIVsmm may or may not be followed by simian AIDS (30). This variability in the course of SIVsmm infection of RMs prompted us to include in the study the group of SIVmac239-infected RMs. We would like to point out that this study did not include a group of SIVmac239infected SMs, since a previous study indicated that this model, in contrast with experimental inoculation with uncloned SIVsmm, does not result in robust virus replication, even in the presence of a weak cellular and humoral antiviral immune response (57).

By combining our kinetics analysis of the SIV-induced changes in the SM and RM transcriptome with ontology profiling, we were able to (a) assess the overall impact of SIV infection on the transcriptome of SMs and RMs; (b) describe the transcriptional profile of acute and chronic SIV infection of SMs, with specific focus on gene families that are involved in the host immune response to the virus; (c) define discrete differences in the SIV-induced changes of gene expression between RMs and SMs; and (d) identify a number of immune regulatory and virus restriction genes whose differential regulation between SMs and RMs makes them promising candidates for further and more focused studies. The observation that the molecular signature of SIVsmm infection in peripheral blood was largely concordant with that of LNs provides a strong validation of the array data in a different and independent set of experiments. In addition, the fact that many features of the transcriptional profile observed during SIVsmm infection of SMs are also present in SIV-infected AGMs $(58,59)$ provided a biological validation of the results of our current study in a different natural host species.

The central finding of the current study is that, in both SMs and RMs, primary SIV infection induces major alterations in the transcriptome, consisting of a vigorous, early, innate, and adaptive immune activation, and upregulation of genes related to cell cycle and apoptosis and genes coding for factors that restrict virus replication. In particular, by profiling genomic expression during primary SIV infection at intervals as early as 3 days after infection, our data demonstrate that SMs exhibit a strong innate immune response to the virus that is equivalent in magnitude to, or even exceeding, that of RMs. These innate immune response genes that are upregulated in SMs include a large number of ISGs whose expression upon SIV infection increased up to 46-fold compared with baseline levels. Importantly, a key difference between the 2 studied species was that SMs resolved the widespread expression of innate effector molecules, including 20 out of 22 ISGs, during the postacute phase of infection (i.e., by day 30 ). In contrast, SIVmac239-infected RMs maintained this elevated expression of ISGs well into the chronic phase of infection. This latter finding is consistent with the observation of high levels of ISG mRNAs in HIV-infected individuals during chronic infection $(60,61)$. Recent data in mice have demonstrated that IFN- $\alpha$ signaling is critical to inducing cell-cycle entry of dormant HSCs; however, HSCs subject to long-term in vivo IFN- $\alpha$ stimulation are functionally compromised and impaired in their regenerative capacity (62). In the context of the current study, the widespread induction of an early IFN- $\alpha$ response observed in both SMs and RMs likely acts (a) to initiate antiviral activities and (b) as a homeostatic response to the massive immunological insult inflicted by the severe deple- 
tion of mucosal $\mathrm{CD} 4^{+} \mathrm{T}$ cells. The work by Essers et al. suggest that the failure of RMs and humans to silence the IFN response in the postacute and chronic phases of SIV/HIV infection may be a direct cause of hematopoietic defects (62). Coincident with the return to baseline expression levels of ISGs and other genes related to adaptive immune activation, cell cycle, and apoptosis, SIVinfected SMs (but not SIV-infected RMs) manifested a marked upregulation of a number of immune regulatory genes. Collectively, these data demonstrate that SMs generate a robust, extensive early immune response to SIV and strongly suggest that the low levels of immune activation observed during chronic infection could result from active immune regulatory mechanisms. Alternatively, or in addition, the lack of ISG upregulation during chronic SIVsmm infection of SMs could be, at least in part, related to a partial refractoriness of pDCs to respond to TLR-7/9 ligands (29). This conclusion is consistent with recent data from us and others demonstrating that SMs experience a significant, but transient, increase in $T$ cell activation during the acute phase of SIV infection $(18,28,31,32)$. In addition, the observation of an early, active regulation of the immune response shown by our genomic analysis in SIV-infected SMs is consistent with the finding that, in these animals, the immune regulatory molecule PD1 is rapidly upregulated in $\mathrm{LN}$-derived $\mathrm{CD}^{+} \mathrm{T}$ cells $(28)$ and supports a model in which active immunoregulatory mechanisms control SIV-induced immune activation (Figure 8F). The examination of our gene list for molecules known to negatively regulate immune activation led to 3 interesting preliminary observations: (a) the gene encoding IDO, an immunosuppressive protein produced by macrophages and DCs that negatively regulates $\mathrm{T}$ lymphocyte proliferation and antagonizes IFN activity (63), was induced in SM, but not RMs, after SIV infection; (b) ADAR mRNA, encoding an adenosine deaminase that suppresses ISG expression and supports hematopoiesis (64), was induced to high levels in SMs, but not RMs, during the acute phase of SIV infection; and (c) the genes encoding TIM3, a recently characterized marker of T cell exhaustion (65), and its ligand, galectin-9, a proapoptotic ISG (66), are upregulated during acute SIV infection of RMs but not SMs. Confirmatory studies will elucidate the potential role of these genes in maintaining the immune-attenuated phenotype shown by chronically SIV-infected SMs.

A recent study by Mandl et al. described reduced (but not absent) in vitro production of IFN- $\alpha$ in response to TLR-7 and - 9 stimulation by pDCs of SMs when compared with the same cells of RMs (29). Based on these results, these authors proposed a model in which the low immune activation of SIV-infected SMs is the consequence of a muted innate immune response to the virus. A prediction of this hypothesis would have been that reduced upregulation of ISGs is present during acute SIV infection of SMs. Our in vivo analysis, however, does not confirm this prediction and instead indicates very clearly that acute SIV infection of SMs is associated with an ISG upregulation that is similar, if not even stronger, than that observed in SIV-infected RMs. This apparent discrepancy could possibly be reconciled by postulating that the early type I IFN response is saturated during acute SIV infection due to high levels of viremia and/or the early upregulation of ISGs in SMs occurs primarily via a TLR-7/9-independent mechanism and/or that, in these animals, additional non-pDC cell types may contribute to the production of type I IFNs during acute SIV infection. While recent data have demonstrated desensitization to IFN- $\alpha / \beta$ signaling in HIV-infected subjects (45), PBMCs from chronically
SIV-infected SMs retained the ability to upregulate ISGs, suggesting that refractoriness to type I IFN does not mediate the postacute downregulation of ISGs. In any event, the current set of data demonstrates unequivocally that a muted IFN- $\alpha$ response during acute infection is neither a feature nor a requirement for reaching a state of nonpathogenicity in SIV-infected SMs.

Of note, we observed no significant increase in transcripts for type I IFNs in blood or LNs, despite comprehensive representation of multiple IFN- $\alpha$ subtypes on the array. This observation is consistent with a recent study in which plasma levels of IFN- $\alpha$ were measured by ELISA in SIV-infected SMs (31) and suggests that upregulation of ISGs represents a much more sensitive indication of the presence of an active type I IFN response in vivo. It is also worth noting that increases in bioactive IFN- $\alpha$ have been detected in plasma from acutely SIV-infected AGMs using bioassays that have an increased sensitivity compared with protein detection by ELISA (56). Importantly, recent immunohistochemistry data have demonstrated that IFN- $\alpha$ can be detected in the LNs of acutely SIVsmm-infected SMs and colocalizes with CD123, suggesting local production of IFN- $\alpha$ by pDCs during the acute phase (J.D. Estes, unpublished observations). This observation is compatible with our finding of a strong ISG induction during acute SIV infection of SMs in both blood and LNs.

In addition to classical ISGs, we also noted extensive upregulation of 2 other classes of genes with direct anti-HIV activity: restriction factors and defensins. The expression of retroviral restriction factors such as TRIM family members $(67,68)$, APOBEC3 proteins (69), and BST2/tetherin (42), has been demonstrated to be type I IFN inducible. In the current set of data, upregulation of mRNA for the majority of restriction factors was observed at days 7-10 after infection in both species but returned to baseline levels by day 14. In comparison, upregulation of classical ISGs remained elevated until day 30 after infection in SMs and even during chronic infection in RMs, suggesting that ISGs and restriction factors are subject to independent pathways of type I IFN regulation. Our analysis also revealed that expression of $\alpha$ and $\theta$ defensins, i.e., 2 classes of molecules with potent inhibitory activity against HIV in vitro (70-72), was increased in LNs from SIV-infected SMs. As we were unable to obtain LN biopsies from SIV-infected RMs at day 30 after infection, it remains possible that RMs may upregulate defensins with kinetics similar to SMs. Collectively, these data indicate that, although SMs upregulate selected defensins and restriction factors during SIV infection, robust viral replication still occurs despite this extensive antiviral response. This apparent paradox may be explained by postulating that defensins and/or restriction factor expression may protect from disease progression in SIV-infected SMs despite chronic viral replication by shielding specific lymphocyte subsets that are vital to immune homeostasis, such as central-memory $\mathrm{CD}^{+}{ }^{+} \mathrm{T}$ cells, from SIV infection.

To define previously unrecognized molecular pathways that may be indicative of chronic immune activation during pathogenic SIVmac239 infection of RMs, we used a systems biology analysis to identify genes with expression profiles significantly correlated with protein expression of $\mathrm{Ki}-67$ in $\mathrm{CD}^{+} \mathrm{T}$ cells, i.e., a key marker of $\mathrm{T}$ cell activation. Among the top-scoring genes were CCR5 and, with an inverse correlation, IL7R. The contribution of both genes to HIV pathogenesis has been well characterized $(73,74)$ and indicates that this approach can accurately identify genes linked to immune activation. Interestingly, other top-scoring molecules were LAG3 and TIM3, which are both markers of $\mathrm{CD}^{+} \mathrm{T}$ cell exhaustion $(53,65)$. 
Further studies will examine the role of these factors in the pathogenesis of SIVmac239-associated immune dysfunction. The observation that CCR5, LAG3, and TIM3 shared similar expression profiles in SIVmac239-infected RMs is quite intriguing and suggests a possible relationship between CCR5 expression (and thus susceptibility of CD $4^{+} \mathrm{T}$ cells to infection) and expression of markers of T cell exhaustion. Of note, CCR5 and LAG3 were found to be overexpressed (and IL7R underexpressed) in "exhausted" $\mathrm{CD} 8^{+} \mathrm{T}$ cells in a recent study of the transcriptional profile of murine LCMV-specific CD8 ${ }^{+} \mathrm{T}$ cells with naive, effector, memory, and exhausted phenotypes (53).

A similar pattern of active innate and adaptive immune response to SIV infection that was then followed by a rapid downmodulation of this response by day 30 to 40 after infection has also been observed in 2 independent longitudinal analyses of the changes in gene expression induced by nonprogressive SIVagm infection of AGMs $(58,59)$. While a number of differences between SMs and AGMs in response to SIV infection may become apparent upon further analysis of the available data sets, the observed similarities, particularly in terms of early, dramatic, and transient upregulation of ISGs, suggest a common evolutionary pathway of disease resistance in natural SIV hosts that relies on the active downmodulation of an otherwise normal innate immune response to the virus. While this possible conclusion should be confirmed in other species of natural SIV hosts, its potential implications are far reaching and include the identification of a protective in vivo role of rapidly tuning down the immune activation occurring during acute SIV infection once it becomes clear that the immune system is not capable of completely suppressing virus replication. Ultimately, it is hoped that further elucidation of the genes and molecular pathways involved in maintenance of a state of low immune activation in chronically SIVinfected SMs and AGMs will provide additional targets for therapeutic interventions aimed at limiting or abrogating the aberrant immune activation that is associated with HIV infection.

\section{Methods}

Animals and SIV infection. As shown in Figure 1A, a total of 18 nonhuman primates were included in this study, of which 17 underwent full analysis. 5 SMs and 5 RMs were inoculated with an uncloned SIVsmm derived from an experimentally infected SM at 11 days after infection ( $1 \mathrm{ml}$ of plasma) as described previously $(18,28)$. One RM was excluded from the analysis due to absence of robust virus replication. In addition, 8 RMs were inoculated i.v. with 3000 TCID50 (50\% tissue culture infectious dose) of SIVmac239. For LNs, biopsies were taken from 2 SIVsmm-infected SMs and 2 SIVmac239-infected RMs for each interval. All animals used in this study were housed at the Yerkes National Primate Research Center in accordance with the regulations of the American Association of Accreditation of Laboratory Animal Care standards. This study was approved by the Institutional Animal Care and Usage Committees of Emory University and the University of Pennsylvania.

Plasma viral load measurement. SIV plasma viral RNA levels were quantitated using real-time PCR as described previously $(22,75)$.

Immunophenotyping. CD4 ${ }^{+} \mathrm{T}$ cell counts were measured using flow cytometry on whole blood according to standard procedures, as described previously (18). mAb clones used (anti-CD4-FITC, L200; anti-CD3-Alexa Fluor 700, SP34-2, Ki-67 FITC, clone B56; anti-CD8 Pacific Blue, Pacific Orange and PE-Texas Red, clone RPA-T8; [BD Biosciences - Pharmingen], anti-CD4 PE-Cy5.5 and Pacific Orange, clone OKT4 [eBioscience]) were designed for detection of human molecules but have been demonstrated to be cross-reactive with SMs and RMs (22). Data acquisition on at least 100,000 events was performed using an LSRII flow cytometer, and data analysis was performed using FlowJo software (Tree Star).
Immunobistochemical staining for defensins. $\alpha$-defensin ${ }^{+}$cells were detected in LN sections of SM at 30 dpi by immunohistochemical staining by previously reported protocols (76). In brief, for epitope retrieval, tissue sections in $10 \mathrm{mM}$ citrate buffer, $\mathrm{pH} 6.0$, were heated for 15 minutes to $95^{\circ} \mathrm{C}$ in a water bath. The sections were then stained by overnight incubation at $4{ }^{\circ} \mathrm{C}$ with a $1: 100$ dilution of mouse mAb against human $\alpha$-defensin 1-3 (clone: DEF3AbD; SeroTec) or a mouse anti-human IgG1 isotype control, and then stained using the DAKO EnVision + System, HRP.

$R N A$ purification. Using standard venipuncture, $2.5 \mathrm{ml}$ of blood was drawn directly into PAXgene blood RNA tubes (BD) and stored at $-80^{\circ} \mathrm{C}$ until purification. Total RNA from blood was purified with the PAXgene blood RNA kits (QIAGEN) according to manufacturer's protocol utilizing on-column DNAse digestion. Total RNA from LNs was purified using previously published procedures $(49,77)$. RNA quantity was estimated using spectrophotometry and/or nanodrop analysis, and integrity was assessed using $2 \%$ agarose gel electrophoresis and Agilent Bioanalyzer electrophoresis. Purification of RNA samples was performed in balanced blocks to minimize experimental bias according to standard microarray experimental design.

Microarray bybridization and bemoglobin blocking. All RNA samples were hybridized to Affymetrix GeneChip Rhesus Macaque Genome Arrays (Affymetrix), which contains 52,024 individual probe sets that assay over 47,000 transcripts. To block nonspecific binding by hemoglobin transcripts, a set of 5 peptide nucleic acid (PNA) oligonucleotides (Bio-Synthesis Inc.) were designed to bind to regions of hemoglobin $\alpha$ and $\beta$ mRNA and block reverse transcription. PNAs were designed to be complementary to consensus regions between the RM and SM hemoglobin mRNAs, which were derived from sequences available at GenBank; de novo sequencing was performed in our laboratory. PNA sequences, concentrations, and hemoglobin targets are available in Supplemental Table 3. Blocking PNAs were added to the first-strand reverse transcription mix with $0.5 \mu \mathrm{g}$ of total RNA, and reverse transcription, amplification, fragmentation, and hybridization were performed using 2-cycle cDNA synthesis as detailed in the Affymetrix GeneChip Expression Analysis Technical Manual. RNA samples were processed by a single operator in balanced blocks to minimize handling bias. Since the Affymetrix arrays were designed for use on cRNAs derived from RMs, we compared hybridization signals between RM and SM samples and observed no substantial difference among the intensity mean, median, and range (Supplemental Figure 1 and Supplemental Table 1).

Microarray data analysis. A detailed description of the methods used for analysis of the microarray data is discussed in the Supplemental Methods. In brief, CEL files from individual hybridizations were normalized using the robust multi-chip average (RMA) algorithm. Normalization and subsequent analyses were performed using Partek Genomics Suite software v6.4 (Partek Inc).

Accession numbers. The microarray data sets were submitted to the GEO repository according to MIAME (Minimum Information About a Microarray Experiment) standards (GEO accession number GSE16147, blood; GEO accession number GSE17626, LNs). Partial cDNA sequences for RM and SM hemoglobin were submitted to GenBank (GenBank accession numbers GQ205391, GQ205392).

Statistics. One-way ANOVA was used to determine significance of probe set intensity changes after SIV infection in SMs $(P=0.0008342)$ and SIVmac239 infection in RMs $(P=0.00746)$ in the peripheral blood data set. $P$ values were adjusted for multiple hypothesis testing using the Benjamini-Hochberg false discovery rate method. Probe sets with significantly differential expression in LNs were defined using the 2-sample Wilcoxon's signed-rank test $(P=0.05)$. Significant $P$ values were used in combination with a 2 -fold up/down criteria to define differentially expressed genes. Significant ontology enrichment was determined using Fisher's exact test (right-tailed; $P=0.05$ ) See Supplemental Methods for detailed descriptions. 


\section{Acknowledgments}

The authors wish to thank Stephanie Ehnert and all the animal care and veterinary staff at the Yerkes National Primate Center as well as Seema Garg and Benton Lawson and the Virology Core of the Emory Center for AIDS Research (CFAR). We are grateful to Francois Villinger of Emory University for providing recombinant SM IFN- $\alpha$. In addition, we are thankful to John Tobias of the Bioinformatics Core of the University of Pennsylvania School of Medicine and to Jean Francois Olivier and Christopher Davies of Affymetrix for their bioinformatics support. We would also like to thank David Banner for technical assistance and Colleen O'Neil and Tim Leonard for help with manuscript preparation. We are also thankful to Mark Feinberg for his discussion. This work was supported by NIH grants AI-66998 and HL-75766 (to G. Silvestri) and AI-048484 (to A.T. Haase); NIH grant RFP NIH-NIAID-DAIT-BAA04-18; the Canadian Institutes of Health Research (200608PAN167150-PAA-ADHD-48072); funding from the Li Ka Shing Foundation (to D.J. Kelvin); and NIH grant P51 RR00165 to the Yerkes
National Primate Research Center. S.E. Bosinger is a recipient of a Canadian Institutes of Health Research HIV/AIDS Research Initiative Fellowship (HFE-85139).

Received for publication June 8, 2009, and accepted in revised form October 19, 2009.

Address correspondence to: Guido Silvestri, Suite 705 StellarChance Laboratories, 422 Curie Boulevard, Philadelphia, Pennsylvania 19104, USA. Phone: (215) 573-5363; Fax: (215) 573-5369; E-mail: gsilvest@mail.med.upenn.edu. Or to: Ashley T. Haase, Department of Microbiology, University of Minnesota, Box 196, 420 Delaware Street S.E., Minneapolis, Minnesota 55455, USA. Phone: (612) 624-4442; Fax: (612) 626-0623; E-mail: haase001@ umn.edu. Or to: David J. Kelvin, Toronto Medical Discovery Tower, Rm 3-916, 101 College Street, Toronto, Ontario M5G 1L7, Canada. Phone: (416) 581-7605; Fax: (416) 581-7606; E-mail: dkelvin@ uhnresearch.ca.
1. Picker, L.J. 2006. Immunopathogenesis of acute AIDS virus infection. Curr. Opin. Immunol. 18:399-405.

2. Mellors, J.W., et al. 1996. Prognosis in HIV-1 infection predicted by the quantity of virus in plasma. Science. 272:1167-1170.

3. Douek, D.C., Roederer, M., and Koup, R.A. 2009. Emerging concepts in the immunopathogenesis of AIDS. Annu. Rev. Med. 60:471-484.

4. Sodora, D.L., and Silvestri, G. 2008. Immune activation and AIDS pathogenesis. AIDS. 22:439-446.

5. Aziz, N., et al. 1999. Stability of plasma levels of cytokines and soluble activation markers in patients with human immunodeficiency virus infection. J. Infect. Dis. 179:843-848.

6. De Boer, R.J., Mohri, H., Ho, D.D., and Perelson, A.S. 2003. Turnover rates of B cells, T cells, and NK cells in simian immunodeficiency virus-infected and uninfected rhesus macaques. J. Immunol. 170:2479-2487.

7. Hellerstein, M., et al. 1999. Directly measured kinetics of circulating $\mathrm{T}$ lymphocytes in normal and HIV-1-infected humans. Nat. Med. 5:83-89.

8. Picker, L.J., et al. 2004. Insufficient production and tissue delivery of CD4+ memory $\mathrm{T}$ cells in rapidly progressive simian immunodeficiency virus infection. J. Exp. Med. 200:1299-1314.

9. Deeks, S.G., et al. 2004. Immune activation set point during early HIV infection predicts subsequent CD4+ T-cell changes independent of viral load. Blood. 104:942-947.

10. Leng, Q., et al. 2001. Immune activation correlates better than HIV plasma viral load with CD4 T-cell decline during HIV infection. J. Acquir. Immune Defic. Syndr. 27:389-397.

11. Sousa, A.E., et al. 2002. CD4 T cell depletion is linked directly to immune activation in the pathogenesis of HIV-1 and HIV-2 but only indirectly to the viral load. J. Immunol. 169:3400-3406.

12. Giorgi, J.V., et al. 2002. Predictive value of immunologic and virologic markers after long or short duration of HIV-1 infection. J. Acquir. Immune Defic. Syndr. 29:346-355.

13. Hazenberg, M.D., et al. 2003. Persistent immune activation in HIV-1 infection is associated with progression to AIDS. AIDS. 17:1881-1888.

14. Giorgi, J.V., et al. 1999. Shorter survival in advanced human immunodeficiency virus type 1 infection is more closely associated with $\mathrm{T}$ lymphocyte activation than with plasma virus burden or virus chemokine coreceptor usage. J. Infect. Dis. 179:859-870.

15. Paiardini, M., Pandrea, I., Apetrei, C., and Silvestri, G. 2009. Lessons Learned from the Natural Hosts of HIV-Related Viruses. Annu. Rev. Med. 60:485-495.

16. Hahn, B.H., Shaw, G.M., De Cock, K.M., and Sharp,
P.M. 2000. AIDS as a zoonosis: scientific and public health implications. Science. 287:607-614.

17. Dunham, R., et al. 2006. The AIDS resistance of naturally SIV-infected sooty mangabeys is independent of cellular immunity to the virus. Blood. 108:209-217.

18. Gordon, S.N., et al. 2007. Severe depletion of mucosal CD4+ T cells in AIDS-free simian immunodeficiency virus-infected sooty mangabeys. J. Immunol. 179:3026-3034.

19. Gordon, S.N., et al. 2008. Short-lived infected cells support virus replication in sooty mangabeys naturally infected with simian immunodeficiency virus: implications for AIDS pathogenesis. J. Virol. 82:3725-3735.

20. Klatt, N.R., et al. 2008. Availability of activated $\mathrm{CD} 4+\mathrm{T}$ cells dictates the level of viremia in naturally SIV-infected sooty mangabeys. J. Clin. Invest. 118:2039-2049.

21. Rey-Cuille, M.A., et al. 1998. Simian immunodeficiency virus replicates to high levels in sooty mangabeys without inducing disease. J. Virol. 72:3872-3886.

22. Silvestri, G., et al. 2003. Nonpathogenic SIV infection of sooty mangabeys is characterized by limited bystander immunopathology despite chronic highlevel viremia. Immunity. 18:441-452.

23. Silvestri, G., et al. 2007. Understanding the benign nature of SIV infection in natural hosts. J. Clin. Invest. 117:3148-3154.

24. Schindler, M., et al. 2006. Nef-mediated suppression of $\mathrm{T}$ cell activation was lost in a lentiviral lineage that gave rise to HIV-1. Cell. 125:1055-1067.

25. Schindler, M., et al. 2008. Inefficient Nef-mediated downmodulation of CD3 and MHC-I correlates with loss of CD4+T cells in natural SIV infection. PLoS Pathog. 4:e1000107.

26. Brenchley, J.M., et al. 2006. Microbial translocation is a cause of systemic immune activation in chronic HIV infection. Nat. Med. 12:1365-1371.

27. Pandrea, I., et al. 2007. Paucity of CD4+CCR5+ T cells is a typical feature of natural SIV hosts. Blood. 109:1069-1076.

28. Estes, J.D., et al. 2008. Early resolution of acute immune activation and induction of PD-1 in SIV-infected sooty mangabeys distinguishes nonpathogenic from pathogenic infection in rhesus macaques. J. Immunol. 180:6798-6807.

29. Mandl, J.N., et al. 2008. Divergent TLR7 and TLR9 signaling and type I interferon production distinguish pathogenic and nonpathogenic AIDS virus infections. Nat. Med. 14:1077-1087.

30. Silvestri, G., et al. 2005. Divergent host responses during primary simian immunodeficiency virus SIVsm infection of natural sooty mangabey and nonnatural rhesus macaque hosts. J. Virol. 79:4043-4054.

31. Meythaler, M., et al. 2009. Differential CD4+ T-lymphocyte apoptosis and bystander T-cell activation in rhesus macaques and sooty mangabeys during acute simian immunodeficiency virus infection. J. Virol. 83:572-583.

32. Muthukumar, A., et al. 2005. Timely triggering of homeostatic mechanisms involved in the regulation of T-cell levels in SIVsm-infected sooty mangabeys. Blood. 106:3839-3845.

33. Dennis, G., Jr., et al. 2003. DAVID: Database for annotation, visualization, and integrated discovery. Genome Biol. 4:P3.

34. Yoneyama, M., et al. 2004. The RNA helicase RIG-I has an essential function in double-stranded RNAinduced innate antiviral responses. Nat. Immunol. 5:730-737.

35. Kawai, T., et al. 2005. IPS-1, an adaptor triggering RIG-I- and Mda5-mediated type I interferon induction. Nat. Immunol. 6:981-988.

36. Freeman, G.J., et al. 2000. Engagement of the PD-1 immunoinhibitory receptor by a novel B7 family member leads to negative regulation of lymphocyte activation. J. Exp. Med. 192:1027-1034.

37. Mellor, A.L., and Munn, D.H. 2004. IDO expression by dendritic cells: tolerance and tryptophan catabolism. Nat. Rev. Immunol. 4:762-774.

38. Cerdan, C., Devilard, E., Xerri, L., and Olive, D. 2001. The C-class chemokine lymphotactin costimulates the apoptosis of human CD4(+) T cells. Blood. 97:2205-2212.

39. Schreiber, V., Dantzer, F., Ame, J.C., and de Murcia, G. 2006. Poly(ADP-ribose): novel functions for an old molecule. Nat. Rev. Mol. Cell Biol. 7:517-528.

40. Zhang, N., Hopkins, K., and He, Y.W. 2008. c-FLIP protects mature $\mathrm{T}$ lymphocytes from TCR-mediated killing. J. Immunol. 181:5368-5373.

41. Kovaleski, B.J., et al. 2006. In vitro characterization of the interaction between HIV-1 gag and human lysyltRNA synthetase. J. Biol. Chem. 281:19449-19456.

42. Neil, S.J., Zang, T., and Bieniasz, P.D. 2008. Tetherin inhibits retrovirus release and is antagonized by HIV-1 Vpu. Nature. 451:425-430.

43. Van Damme, N., et al. 2008. The interferoninduced protein BST-2/CD317 restricts release of virions from infected cells and is down-regulated from the cell surface by HIV-1 Vpu. Cell Host Microbe. 3:245-252.

44. Sadler, A.J., and Williams, B.R. 2008. Interferoninducible antiviral effectors. Nat. Rev. Immunol. 8:559-568.

45. Hardy, G.A., et al. 2009. Desensitization to type I interferon in HIV-1 infection correlates with mark- 
ers of immune activation and disease progression. Blood. 113:5497-5505.

46. Embretson, J., et al. 1993. Massive covert infection of helper T lymphocytes and macrophages by HIV during the incubation period of AIDS. Nature. 362:359-362.

47. Pantaleo, G., et al. 1993. HIV infection is active and progressive in lymphoid tissue during the clinically latent stage of disease. Nature. 362:355-358.

48. Reimann, K.A., et al. 1994. Immunopathogenic events in acute infection of rhesus monkeys with simian immunodeficiency virus of macaques. J. Virol. 68:2362-2370.

49. Li, Q., et al. 2009. Microarray analysis of lymphatic tissue reveals stage-specific, gene expression signatures in HIV-1 infection. J. Immunol. 183:1975-1982.

50. Ganz, T. 2002. Immunology. Versatile defensins. Science. 298:977-979.

51. Douek, D.C. 2003. Disrupting T-cell homeostasis: how HIV-1 infection causes disease. AIDS Rev. 5:172-177.

52. Querec, T.D., et al. 2009. Systems biology approach predicts immunogenicity of the yellow fever vaccine in humans. Nat. Immunol. 10:116-125.

53. Wherry, E.J., et al. 2007. Molecular signature of CD8+ $\mathrm{T}$ cell exhaustion during chronic viral infection. Immunity. 27:670-684.

54. Pandrea, I.V., et al. 2007. Acute loss of intestinal $\mathrm{CD} 4+\mathrm{T}$ cells is not predictive of simian immunodeficiency virus virulence. J. Immunol. 179:3035-3046.

55. Cumont, M.C., et al. 2008. Early divergence in lymphoid tissue apoptosis between pathogenic and nonpathogenic simian immunodeficiency virus infections of nonhuman primates. J. Virol. 82:1175-1184.

56. Diop, O.M., et al. 2008. Plasmacytoid dendritic cell dynamics and alpha interferon production during Simian immunodeficiency virus infection with a nonpathogenic outcome. J. Virol. 82:5145-5152.

57. Kaur, A., et al. 1998. Diverse host responses and outcomes following simian immunodeficiency virus SIVmac239 infection in sooty mangabeys and rhesus macaques. J. Virol. 72:9597-9611.

58. Jacquelin, B., et al. 2009. Nonpathogenic SIV infection of African green monkeys induces a strong but rapidly controlled type I IFN response. J. Clin. Invest. 119:3544-3555.

59. Lederer, S., et al. 2009. Transcriptional profiling in pathogenic and non-pathogenic SIV infections reveals significant distinctions in kinetics and tissue compartmentalization. PLoS Pathog. 5:e1000296.

60. Hyrcza, M.D., et al. 2007. Distinct transcriptional profiles in ex vivo CD4+ and CD8+ T cells are established early in human immunodeficiency virus type 1 infection and are characterized by a chronic interferon response as well as extensive transcriptional changes in CD8+ T cells. J. Virol. 81:3477-3486.

61. Sedaghat, A.R., et al. 2008. Chronic CD4+ T-cell activation and depletion in human immunodeficiency virus type 1 infection: type I interferonmediated disruption of T-cell dynamics. J. Virol. 82:1870-1883.

62. Essers, M.A., et al. 2009. IFNalpha activates dormant haematopoietic stem cells in vivo. Nature. 458:904-908.

63. Boasso, A., and Shearer, G.M. 2007. How does indoleamine 2,3-dioxygenase contribute to HIV mediated immune dysregulation. Curr. Drug Metab. 8:217-223.

64. Hartner, J.C., Walkley, C.R., Lu, J., and Orkin, S.H. 2009. ADAR1 is essential for the maintenance of hematopoiesis and suppression of interferon signaling. Nat. Immunol. 10:109-115.

65. Jones, R.B., et al. 2008. Tim-3 expression defines a novel population of dysfunctional $\mathrm{T}$ cells with highly elevated frequencies in progressive HIV-1 infection. J. Exp. Med. 205:2763-2779.

66. Chawla-Sarkar, M., et al. 2003. Apoptosis and interferons: role of interferon-stimulated genes as mediators of apoptosis. Apoptosis. 8:237-249.

67. Carthagena, L., et al. 2009. Human TRIM gene expression in response to interferons. PLOS ONE. 4:e4894.

68. Rajsbaum, R., Stoye, J.P., and O'Garra, A. 2008. Type I interferon-dependent and -independent expression of tripartite motif proteins in immune cells. Eur. J. Immunol. 38:619-630.

69. Peng, G., et al. 2006. Induction of APOBEC3 family proteins, a defensive maneuver underlying interferon-induced anti-HIV-1 activity. J. Exp. Med. 203:41-46.

70. Zhang, L., et al. 2002. Contribution of human alpha-defensin 1,2, and 3 to the anti-HIV-1 activity of CD8 antiviral factor. Science. 298:995-1000.

71. Munk, C., et al. 2003. The theta-defensin, retrocyclin, inhibits HIV-1 entry. AIDS Res. Hum. Retroviruses. 19:875-881.

72. Wang, W., et al. 2004. Activity of alpha- and theta-defensins against primary isolates of HIV-1. J. Immunol. 173:515-520.

73. Lederman, M.M., Penn-Nicholson, A., Cho, M and Mosier, D. 2006. Biology of CCR5 and its role in HIV infection and treatment. JAMA. 296:815-826.

74. Leone, A., Picker, L.J., and Sodora, D.L. 2009. IL-2, IL-7 and IL-15 as immuno-modulators during SIV/HIV vaccination and treatment. Curr. HIV Res. 7:83-90.

75. Garber, D.A., et al. 2004. Blockade of T cell costimulation reveals interrelated actions of CD4+ and CD8+ T cells in control of SIV replication. J. Clin. Invest. 113:836-845.

76. Li, Q., et al. 2005. Peak SIV replication in resting memory CD4+ T cells depletes gut lamina propria CD4+ T cells. Nature. 434:1148-1152.

77. Li, Q., et al. 2004. Functional genomic analysis of the response of HIV-1-infected lymphatic tissue to antiretroviral therapy. J. Infect. Dis. 189:572-582. 